\title{
Competence in bronchoscopic treatments in emphysema
}

\author{
Michela BEZZI ${ }^{1}$, Valentina LUZZI $2 *$, Mauro NOVALI 1 , Andrea COMEL ${ }^{3}$, \\ Guido POLESE ${ }^{4}$, Lorenzo CORBETTA 5
}

${ }^{1}$ Spedali Civili di Brescia Hospital, Brescia, Italy; ${ }^{2}$ Department of Interventional Pneumology, Careggi University Hospital, Florence, Italy; ${ }^{3}$ Operative Unit of Pneumology, Department of Medicine, P. Pederzoli Hospital, Peschiera del Garda, Verona, Italy; ${ }^{4}$ Operative Unit of Pneumology, Hospital of Villafranca, Villafranca di Verona Centro Polivalente (CUBO), Villafranca di Verona, Italy; 5 Unit of Interventional Pneumology, University of Florence, Florence, Italy

*Corresponding author: Valentina Luzzi, Department of Interventional Pneumology, Careggi University Hospital, Florence, Italy. E-mail: valentinaluzzi@hotmail.com

\section{A B S T R A C T}

Bronchoscopic lung volume reduction (BLVR) has been proven to be effective in patients with severe emphysema. These techniques are divided into two groups: non-blocking devices that are independent of collateral ventilation and blocking devices that are dependent on collateral ventilation so the choice of the target lobe with inadequate scissors is crucial for the success of the treatment. Current evidences suggest that not all classes and phenotypes of emphysema will benefit from BLVR, and that each technique appears to provide a greater benefit to specific subgroups of patients. Careful patient selection is imperative to prevent insertion in patients unlikely to gain clinical benefits as well as wasteful expenditure. The Chartis system represents the gold standard for measuring fissure integrity and is a direct measurement method. Indirect method is instead the TC study which, thanks to the development of software for quantitative analysis, allows us to obtain reliable measurements of regional density of parenchyma, airway thickness and scissor integrity. BLVR is a highly complex procedure: a first-level competence is a pre-requisite for admission to training. The practical training must be based on discussion of clinical cases and the insertion techniques of the different devices on plastic or animal models, or on cadavers. A specific course, offering final certification, has been developed on the use of Zephyr valves.

(Cite this article as: Bezzi M, Luzzi V, Novali M, Comel A, Polese G, Corbetta L. Competence in bronchoscopic treatments in emphysema. Panminerva Med 2019;61:401-21. DOI: 10.23736/S0031-0808.18.03571-1)

KEY WORDS: Bronchoscopy - Pulmonary emphysema - Pulmonary disease, chronic obstructive.

$\mathrm{T}$ The Global Initiative for Chronic Obstructive Pulmonary Disease (GOLD) defines chronic obstructive pulmonary disease (COPD) as a preventable and treatable disease characterized by progressive airflow limitation that is not fully reversible. The abnormalities are a result of a mixture of small airways disease (obstructive bronchiolitis) and parenchymal destruction (emphysema). The physiologic consequences are a reduction in lung elastic recoil, airflow limitation, air-trapping and hyperinflation. The hyperinflation leads to progressive breathlessness with physical activity and decreased quality of life. As the disease progresses, increased frequency and severity of exacerbations are expected. Ultimately, the disease results in disability and premature mortality.

COPD includes both emphysema and chronic bron- chitis. It is classified according to the severity of airflow obstruction as measured by the post-bronchodilator forced expiratory volume in 1 second (FEV1). Staging is described as mild (Stage I), moderate (Stage II), severe (Stage III), and very severe (Stage IV). ${ }^{1}$

Most risk factors for COPD are environmental. The most common cause of COPD is exposure to tobacco smoke, including second-hand (passive) smoking. Exposure to indoor and outdoor air pollution, occupational exposure to dust, particulates, or toxic vapors or fumes can also cause COPD. Recurrent lower respiratory tract infections during childhood can also increase susceptibility to COPD.

The goals of COPD management include the following: symptom relief, prevention of disease progression, improvement of exercise tolerance and health status (i.e. 
quality of life), prevention and treatment of complications and exacerbations, reduction of mortality. For those with more advanced disease, the most critical goals are to reduce the disability associated with COPD and to improve health status. Current guidelines for the management of COPD, including emphysema, begin with the identification and removal, where possible, of risk factors. Risk factors include tobacco smoking and occupational or environmental exposure to particulates and/or harmful gasses. Most often the diagnosis occurs after the disease is wellestablished and the patient is symptomatic. As COPD progresses, medical therapy with one or more bronchodilator is prescribed. In moderate through very severe COPD, pulmonary rehabilitation including exercise training is recommended to improve symptoms and exercise tolerance. Inhaled corticosteroids may be used in patients with more advanced disease and repeated exacerbations, but its use has been associated with an increased risk of pneumonia. Long-term oxygen therapy in hypoxiemic patients has been shown to improve exercise tolerance and to increase survival. Surgical approaches include lung volume reduction surgery (LVRS) and lung transplantation. ${ }^{2}$

Surgical therapy in the form of LVRS improves symptoms, exercise tolerance and quality of life beyond improvements seen with optimized medical therapy including pulmonary rehabilitation. However, the procedure is characterized by a short-term increase in mortality, significant morbidity, and may involve lengthy postoperative care. LVRS can be considered in the case of advanced pulmonary emphysema. ${ }^{3}$ Surgical resection of hyperinflated portions of the lungs improves the mechanics of respiration. The National Emphysema Treatment Trial (NETT), published in 2003, showed that LVRS achieved significant improvements in exercise capacity and quality of life in patients with predominantly upper-lobe emphysema. ${ }^{4}$ However, postoperative mortality was high $-7.9 \%$ after 90 days. This prompted the development of minimally invasive lung volume reduction procedures with the goal of reducing peri interventional morbidity and mortality.

Therefore, a medical need exists for a minimally invasive approach (such as bronchoscopic LVR, BLVR) that can offer significant efficacy, safety profile (i.e. reduction in the frequency and severity of complications) and that provides meaningful benefits to COPD patients with emphysema ${ }^{5}$ while decreasing mortality and morbidity.

During the past 15 years, different techniques for bronchoscopic lung volume reduction (BLVR) have been developed with the aim of reducing lung hyperinflation yet at the same time avoiding the morbidity and mortality connected with LVRS as well as widening the indications to patients with severe comorbidities. These techniques differ in mechanism of action and reversibility, and their application is dependent on the emphysema distribution and interlobar collateral ventilation. They are divided into two groups: blocking and nonblocking devices and some of them have recently been added in the guidelines of the GOLD as part of the non-pharmacological treatment of stable COPD. The blocking technique is reversible, the latter is not.

The most widely investigated method is valve-BLVR, which is also the only blocking technique. Extensive studies, including several randomized controlled trials (RCT), confirmed the efficacy of this method in a well selected population of emphysema patients. All other BLVR techniques are non-blocking and include lung volume reduction using coils (LVR-COILS), bronchoscopic thermal vapor ablation (BTVA), polymeric lung volume reduction (PLVR) and targeted lung denervation (TLD). Safety, feasibility, and efficacy of these techniques have also been examined in different RCTs, but data are still limited.6,7

\section{Knowledge of the disease: patient selection}

The proper patient and emphysema phenotype selection is the most important criteria. Before deciding on a potential BLVR treatment for patients with COPD, the disease must have first been treated according to the current COPD treatment guidelines, including assessing and insuring the patient's non-smoking status, optimal inhalation therapy, vaccinations, long-term oxygen when appropriate, and pulmonary reha-bilitation. Furthermore, potential surgical treatment options, such as bullectomy and LVRS, have to be considered as treatment options. Candidate patients for BLVR should be discussed in a multidisciplinary team (MDT) meeting to ensure that the best treatment decisions are made. The MDT should be organized in such a way that expert knowledge is available for COPD, chest radiology, surgical options and interventional pulmonology.

A careful review of comorbidities and contraindications is also an important factor before deciding on BLVR (see Table I for guidance on inclusion and exclusion criteria for BLVR). Once this stage has been cleared, the confirmation of pulmonary function in the presence of hyperinflation with RV.

Potential evaluable patients for advanced emphysema treatments are those who are already receiving the best medical treatment, i.e. optimal pharmacological therapy 
mostly with bronchodilators, inhaled corticosteroids, and sometimes maintenance systemic therapies. Patients should also have completed pulmonary rehabilitation and have definitely stopped smoking.

Key parameters are evidence of severe airflow obstruction (forced expiratory volume in the first second [FEV1] $\leq 45 \%$ predicted) and the presence of severe hyperinflation. Hyperinflation should be measured by body plethysmography. Patients with a residual volume (RV) $>200 \%$ predicted are deemed the best responders. In some situations, patients with RV between 175 and $200 \%$ predicted may be considered for treatment if other measures of hyperinflation are favorable, i.e. $\mathrm{RV} / \mathrm{TLC}$ ratio $\geq 0.58 .{ }^{8}$

Patients should be highly symptomatic with respect to dyspnea (modified British Medical Research Council $[\mathrm{mMRC}]>1)$ and restricted in their exercise performance (6-minute walking distance $<450 \mathrm{~m}$ ). However, if a patient has significant symptoms of concurrent chronic airways disease, such as frequent cough, severe bronchial hyperresponsiveness, sputum production and/or frequent exacerbations, the patient should not be considered for BLVR. Also, patients with a very low exercise tolerance (6-minute walking distance $<140 \mathrm{~m}$ ) may not represent the ideal candidates for therapy (See inclusion and exclusion criteria).

Inclusion criteria

- Diagnosis of emphysema COPD;

- subjects of both genders of at least 18 years of age;

- understand and voluntarily sign a patient informed consent form;

- diagnosis of COPD with a $15 \%$ predicted $\leq \mathrm{FEV}_{1} \leq 50 \%$ pred post BD despite optimal medical management;

- TLC $>100 \%$ predicted, $R V \geq 175 \%$ predicted;

- $6 \mathrm{MWT} \geq 140$ meters;

- non-smoker $>8$ weeks prior to signing the informed consent.

\section{Exclusion criteria}

- Evidence of active pulmonary infection;

- history of more than 3 exacerbations with hospitalizations over the past 12 months;

- evidence of severe pulmonary hypertension (sPAP $>45$ $\mathrm{mmHg}$ );

- myocardial infarction or other relevant cardiovascular events in the past 6 months;

- evidence of severe bronchiectasis with greater than two tablespoons of sputum production/day;

- prior BLVR or LVRS procedure;
- prior thoracic surgery;

- pulmonary nodule requiring follow-up within the target lobe.

\section{Radiological assessment}

Current evidences suggest that not all classes and phenotypes of emphysema will benefit from BLVR, and that each technique appears to provide a greater benefit to specific sub-groups of patients. Careful patient selection is imperative to prevent insertion in patients unlikely to gain clinical benefits as well as wasteful expenditure.

High-resolution computed tomography (HRCT) is an absolute prerequisite for adequate patient selection. Normally used in a low-dose protocol with a slice thickness of $1 \mathrm{~mm}$, the HRCT enables the detection and quantification of the destruction of the peripheral lung tissue.

The physician should carefully review the HRCT scan for findings, such as bulla, pathologies or nodules (requiring further assessment and/or follow-up), infiltrations or cavity (suggesting active infection, which should be treated before procedure), or disqualifying criteria (such as severe bronchiectasis, severe paraseptal emphysema, extensive fibrosis or other conditions that may significantly impair outcomes (Figure 1).

When detecting fissure integrity, it is proposed that, if $>90 \%$ of the fissure could be visualized on at least one axis (sagittal, axial, or coronal view), the fissure should be considered complete, implying the absence of significant collateral ventilation between the adjoining lobes. However, outside the experienced radiology core lab setting, visual estimation of fissure completeness has been associated with a rather poor interobserver agreement.

Several computerized semiautomated methods to quantify the completeness of fissures have been developed, which claim to reduce the interobserver variability or

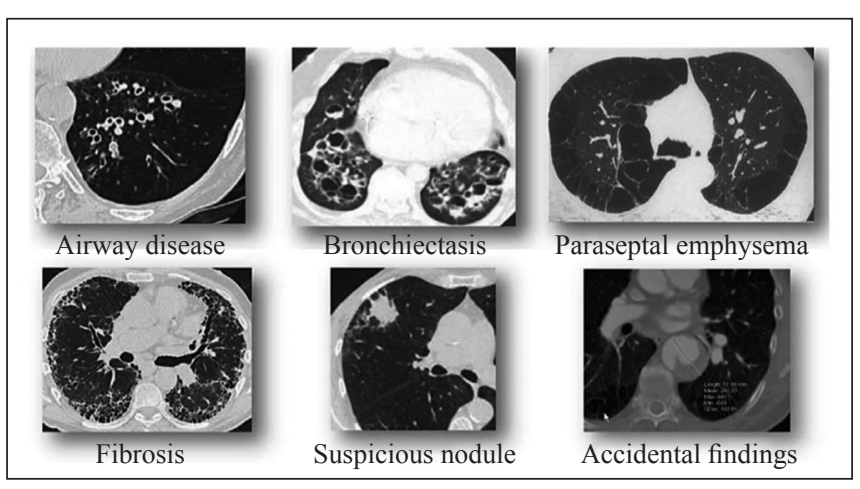

Figure 1.- $\mathrm{CT}$ analysis revealing emphysema morphology not recommended for treatment. 
complement the visual read of an experienced radiologist. These methods are more efficient and have an improved reliability over visual methods. Automated software programs help also to measure the severity and distribution of the emphysema.

Three major selection criteria for BLVR with quantitative analysis of HRCT have been traditionally used: lowattenuation area percentage (LAA\%), heterogeneity score (HS), and fissure integrity (FI) score. LAA\% and HS are important variables to filter subjects with homogeneous and mild to moderate emphysema.

A complete fissure on HRCT is a surrogate for the absence of collateral ventilation, which is defined as the ventilation of alveolar structures through passages that bypass the normal airway. FI is critical for selecting candidates for BLVR. ${ }^{9}$

There are several methods to measure the presence of collateral ventilation. As previously mentioned, an indirect assessment of collateral ventilation is anatomical fissure analysis using quantitative measure of computed tomography $(\mathrm{CT})$.

Anyway, a well-studied direct measurement of collateral airflow is the Chartis system ${ }^{\circledR}$ (Pulmonx, Redwood City, CA, USA), which is an endobronchial pulmonary assessment system 6,7 and that is considered the gold standard . However, performing this measurement requires a bronchoscopy and can include procedural challenges.

The most known software for the quantitative analysis of the CT scan are StratX (Thirona BV, Nijmegen, The Netherlands) (Figure 2, 3).

All quantitative measurements are extracted from the baseline CT scans and automatically computed.

For VIDA an ad hoc FI cut off value of $90 \%$ is currently adopted in the field to separate subjects with complete fissures from the subjects with incomplete fissures.

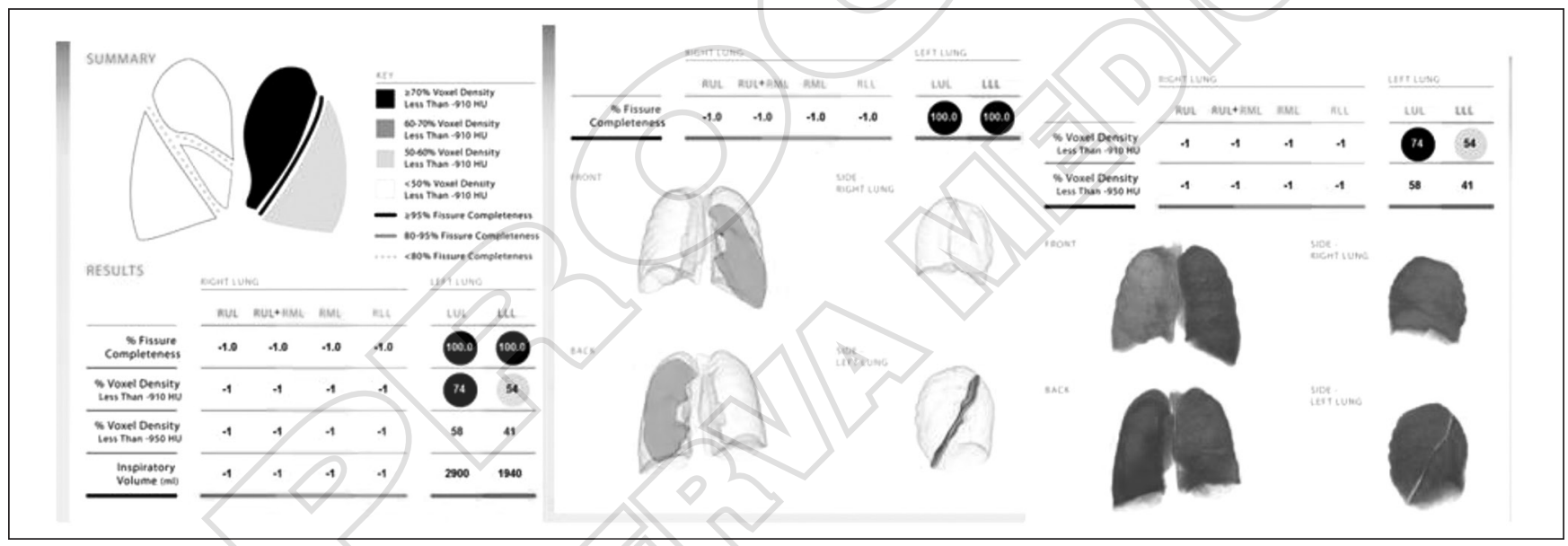

Figure 2.- StratX quantitative analysis of HRCT.

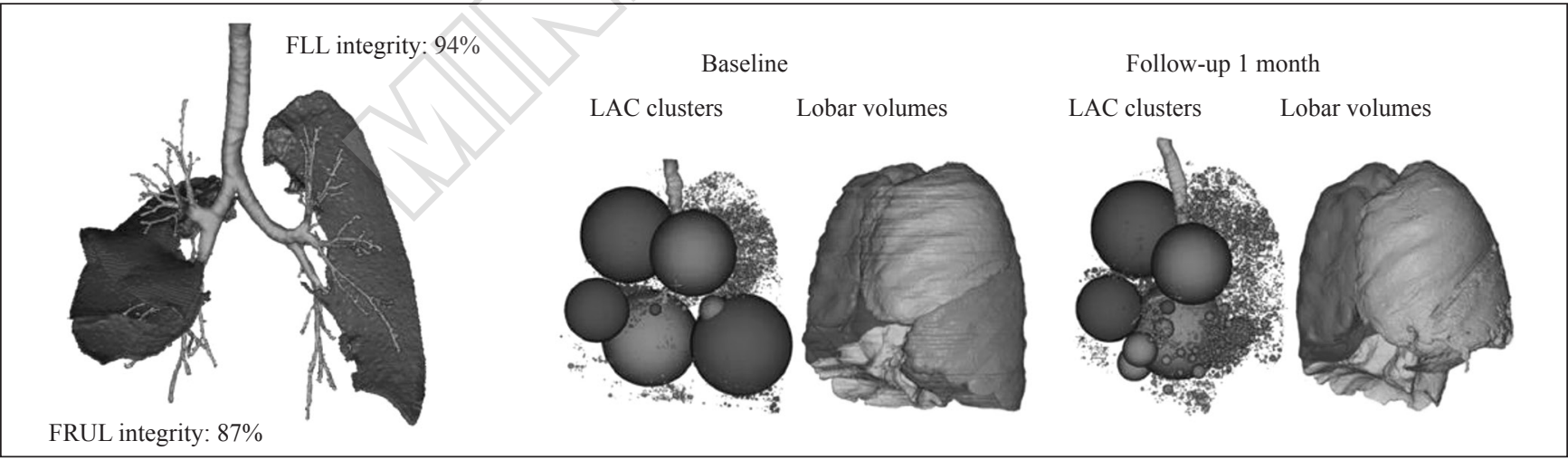

Figure 3.-Automated software analysis for Fissure integrity and emphysema severity and distribution using Apollo software. 
StratX is based on Thirona LungQ version 1.0.0 to assess fissure completeness and lobar tissue destruction at baseline for each subject. In each scan, the lungs, pulmonary fissures, and pulmonary lobes were automatically segmented, visually checked, and edited by trained medical analysts. Each scan was read by two medical analysts, with the first analyst editing the segmentation results where needed and the second analyst checking the results. Based on the results of the lobe and fissure segmentations, a Fissure Completeness Score (FCS) was computed for each lobe as the percentage of the lobar boundaries defined by a fissure.

The application of FCS thresholds results in three groups: complete fissures (FCS $>95 \%$, optimal threshold), incomplete fissures (FCS $<80 \%$ lower threshold), and partially complete fissures (optimal threshold FCS lower threshold). In the partially complete fissure group, where sensitivity and specificity of Quantitative CT (QCT) are suboptimal, the use of Chartis for decision to treat is analyzed. 10

After CT review and assess collateral flow using the Chartis system, patients who have no collateral flow are then treated using a lobar occlusion approach with oneway endobronchial valves.

Patients who have an incomplete fissure at $\mathrm{CT}$ analysis and those with Chartis system-proven collateral flow can be treated with lung volume reduction coil (LVRC) when the tissue destruction is mild to moderately severe and the best emphysema phenotype for coil treatment is centrilobular to moderate panlobular.

When the tissue destruction is too great to allow the use of coils, patients can be treated with one of the more experimental flow-independent techniques, such as a sealant or thermal vapor.

\section{Knowledge of instruments and devices}

Mechanisms of action

Valves

Bronchoscopic placement of valve aims to block the (sub) segmental airways of the most diseased parts of the emphysematous lungs. Successful blockage should result in a full lobar atelectasis and subsequently volume reduction of the treated part of the lung, thereby mimicking lung volume reduction surgery.

Before placing the valves, a clear treatment plan should be developed, starting with the selection of the target lobe and then considering the anatomy of the airways leading to that lobe.
The target lobe should be selected combining absence of collateral ventilation, greater emphysema tissue destruction on $\mathrm{CT}$, and confirmation of low lobar perfusion using perfusion scintigraphy. Also, in patients where multiple target lobes are identified, perfusion scintigraphy, especially in homogeneous emphysema, may be helpful to identify the target lobe (low perfusion in the target, with high perfusion in the ipsilateral, not to be treated lobe). Furthermore, absence of large bulla adjacent to the target lobe, paraseptal emphysema, as well as absence of severe scarring, fibrotic lesions, and significant pleural adhesions is critical. Efficient placement of the valves requires careful airway evaluation and systematic and thoughtful placement. It is important to review the patient's CT scan prior to the procedure to evaluate the airway anatomy and consider the depth and size of each target segment. This will help define the order of valve placement and prevent overlap of the proximal ends of the valves, which can sometimes obstruct access to subsequent airways where valves are to be placed.

The first multicenter, prospective, randomized controlled clinical trial of the Zephyr ${ }^{\circledR}$ Endobronchial Valve $\mathrm{EBV}^{\circledR}$ treatment in patients with heterogeneous emphysema distribution and little to no collateral ventilation, demonstrates significant clinically meaningful benefits over current standard of care medical therapy in lung function, dyspnea, exercise capacity, and quality of life out to at least 12 -months post-procedure. ${ }^{3}$

In January 2018 Zephyr $^{\circledR}$ Endobronchial Valve EBV ${ }^{\circledR}$ had a Premarket Approval (PMA) application with the US Food and Drug Administration (FDA). Three previous randomized controlled trials of the Zephyr EBV (Transform, Impact and Stelvio) in addition to LIBERATE, provide a robust body of evidence showing that the treatment improves breathing, exercise capacity and quality of life, while demonstrating long-term safety, in emphysema patients with little or no collateral ventilation as assessed with the Chartis System. ${ }^{11}, 12$

Concerning IBV Spiration valve, it is currently on going EMPROVE trial that is a randomized, controlled study designed to demonstrate the ability of the Spiration valve System (SVS) to be safety deployed in selected airway of human subjects and to improve lung function.

\section{Coils}

Lung volume reduction coil treatment aims to lead a contraction of the most destructed lung parenchyma by the coils reduces airflow to treated portions of the lung allowing enhanced airflow to healthier untreated portions of the 
lung. This contraction also reduces hyperinflation, which possibly improves diaphragmatic efficiency. Additionally, by gathering up the loose parenchyma of the most severely emphysematous segments, the coil may restore elasticity and recoil to the whole lung, further improving expiratory flow rates and lessening small airway collapse with air trapping.

Centrilobular to moderate panlobular emphysema is the best emphysema phenotype for coil treatment. Severe panlobular emphysema, giant bullae and paraseptal emphysema are morphological patterns of emphysema that are not suitable for treatment with endobronchial coils. Computerized quantitative CT analysis using densitometry should be utilized to more accurately assess the amount of parenchymal destruction and guide optimal selection of the treatment site. Potential sites of treatment are where there is lobar destruction of $20-80 \%$ at the -950 HU threshold on a low (or "soft") kernel reconstructed thin-slice $(1 \mathrm{~mm})$ high-resolution CT. The lobes with the greatest degree of destruction should be targeted. Endobronchial coils can be used independent of collateral ventilation, and, hence, fissure integrity is not a crucial assessment. Furthermore, emphysema patients with both a homogeneous or heterogeneous emphysema distribution can be treated. Similarly, both upper lobe or lower lobe emphysema may be treated. ${ }^{13}$

\section{Aeriseal}

The Aeriseal System is intended to reduce lung volume in order to improve lung function and quality of life in patients with advanced emphysema. It functions by physically occluding both small airways and collateral ait channels causing the treated area to collapse via absorption atelectasis. It is a single use device intended to be used by pulmonologist and thoracic surgeons in a bronchoscopy suite or operating room.

Vapor

Bronchoscopic thermal vapor ablation (Uptake Medical Technology, Inc., Seattle, WA, USA) is currently intended for bilateral treatment of patients with severe upper lobepredominant emphysema irrespective of the interlobar CV. By instillation of heated water vapor, a local inflammatory reaction is induced leading to volume and mass reduction of emphysematous segments.

It is currently the only technique where no implants are left in situ. Furthermore, the segmental application of heated water vapor allows a targeted therapy of emphysema- tous destroyed lung segments and thus BTVA has the potential to manage intralobar heterogeneous emphysema. ${ }^{14}$

Since the mechanism of action for reduction with vapor does not rely upon collapse through blocking or valving of the airway, complete fissures are not required for reduction. Incomplete fissures allow for collateral ventilation between lobes and patients with collateral ventilation are not recommended by valve manufactures due to their inability to achieve lung volume reduction.

The devices

Valves

Two types of valve got CE marking (Conformité Européenne): endobronchial Zephyr valve (Zephyr EBV, Pulmonx, Inc. Palo Alto, CA, USA, Figure 4) and intrabronchial Spiration valve (Spiration IBV, Olympus Respiratory America, Redmond, WA, USA, Figure 5).

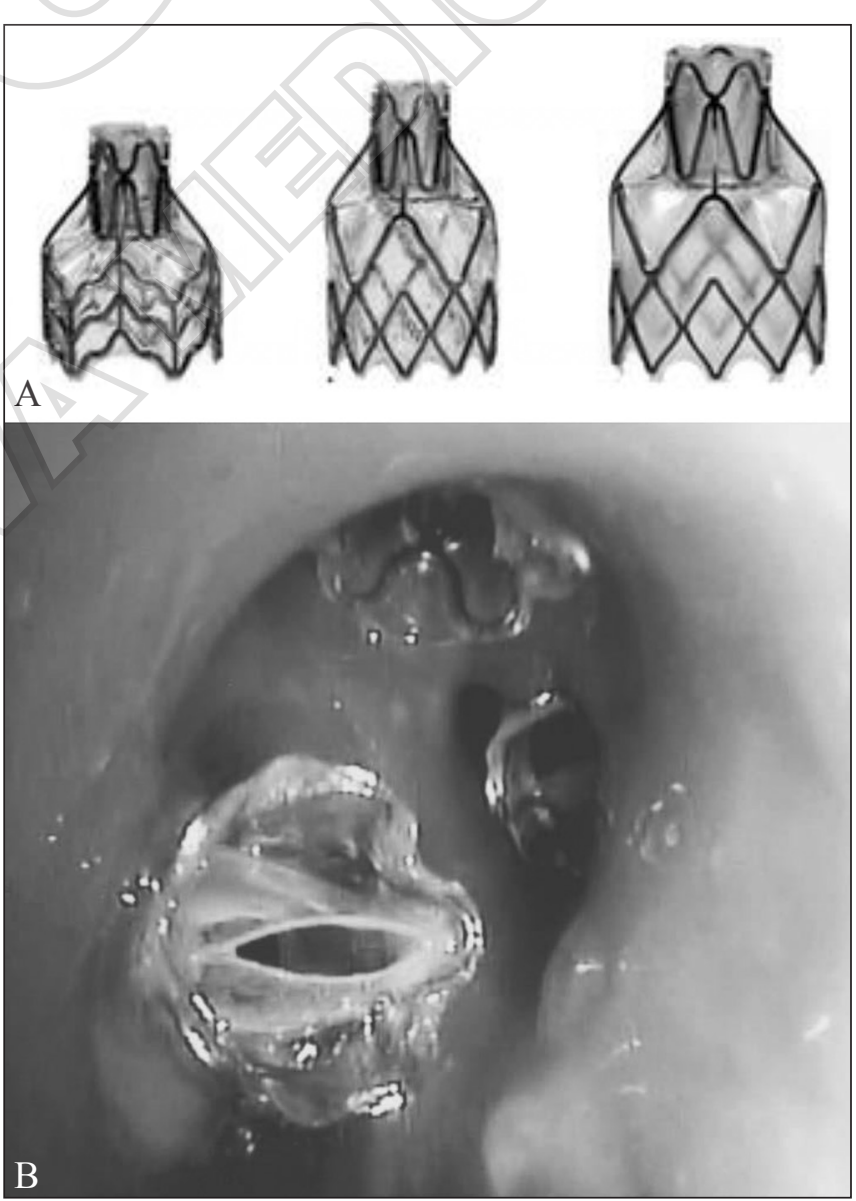

Figure 4.-Pulmonx ${ }^{\circledR}$ Zephyr endobronchial valves (EBV); B) EBV valves. 


\section{Zephyr EBV}

The Zephyr ${ }^{\circledR}$ Endobronchial Valve (Zephyr Valve) is an endobronchial implant designed to occlude airflow in the target lobe and thereby reduce lung volume. The device consists of a oneway silicone duckbill valve attached to a nickel-titanium (nitinol) self-expanding retainer that is covered with a silicone membrane.

Three different size are available (Table I).

It is implanted in the target bronchus during a bronchoscopy procedure using the Zephyr Endobronchial delivery catheter (EDC) that is guided to the targeted bronchus by inserting it through the working channel of an adult bronchoscope.

Successful lobar occlusion may require placement of multiple Zephyr Valves in bronchi of the target lobe. The Zephyr valves permit air to vent out of the treated lobe during exhalation but do not permit refilling of this region during inhalation to reduce hyperinflation due to emphysema. Trapped air in the diseased lobe escapes through the Zephyr Valves until the lung volume of the treated lobe is reduced.

The Zephyr Valve is designed to be a permanent implant, but can be removed, if necessary (Figure 4B).

\section{Spiration IBV}

The valve is comprised of a frame made from Nitinol and a polymer membrane. The membrane is held against the airway mucosa by 6 elastic struts and will expand and contract with airway movement key.

The 5 anchors have tips that penetrate the airway wall to a controlled depth, preventing the valve from migrating. The valve is available in 5-, 6-, and 7- and 9-mm diameters. The valve can be removed by grasping the removal rod with flexible bronchoscopy forceps.

The valve is provided sterile in a disposable loading tool that allows the operator to insert the valve into the distal tip of the catheter. The deployment catheter can be passed through a flexible bronchoscope working channel with a diameter $>2.6 \mathrm{~mm}$. After loading, the catheter is advanced through the bronchoscope working channel to the target implant site. The 5-, 6-, and 7-mm catheters include a feature, the valve deployment guide (VDG), to aid the operator in

TABLE I.-Zephyr endobronchial valve.

\begin{tabular}{llc}
\hline Item number & \multicolumn{1}{c}{ Description } & $\begin{array}{c}\text { Airway diameter } \\
\text { range }\end{array}$ \\
\hline EBD-TS-4.0 & Zephyr 4.0 Endobronchial Valve & $4.0-7.0 \mathrm{~mm}$ \\
EBD-TS-4.0-LP & Zephyr 4.0-LP Endobronchial Valve & $4.0-7.0 \mathrm{~mm}$ \\
EBD-TS-5.5 & Zephyr 5.5 Endobronchial Valve & $5.5-8.5 \mathrm{~mm}$ \\
\hline
\end{tabular}

identifying the location of the proximal end of the valve struts when compressed in the catheter. This feature is a mark on the distal outer surface of the catheter that is visible to the operator via the bronchoscope viewing system. The VDG is in addition to the standard visualization of the compressed valve's membrane struts inside the catheter. The valve is deployed when the operator actuates the deployment handle of the catheter, retracting the catheter sheath to release IBV Valve System Tray Label the valve (Figure 5B).

\section{Coils}

The coil is delivered through a standard $2.8 \mathrm{~mm}$ inner diameter therapeutic bronchoscope. The coil system is a two-part system that consists of: 1) sterile implants ( 3 sizes: $100 \mathrm{~mm}, 125 \mathrm{~mm}$, and $150 \mathrm{~mm}$ );2) sterile, disposable, single-use (single-patient) delivery system consisting of a cartridge, a catheter, a guidewire and forceps (Figure 6).

Each coil implantation procedure must be performed under fluoroscopy in accordance with the instruction for use (IFU). Use of general anesthesia or conscious sedation is at

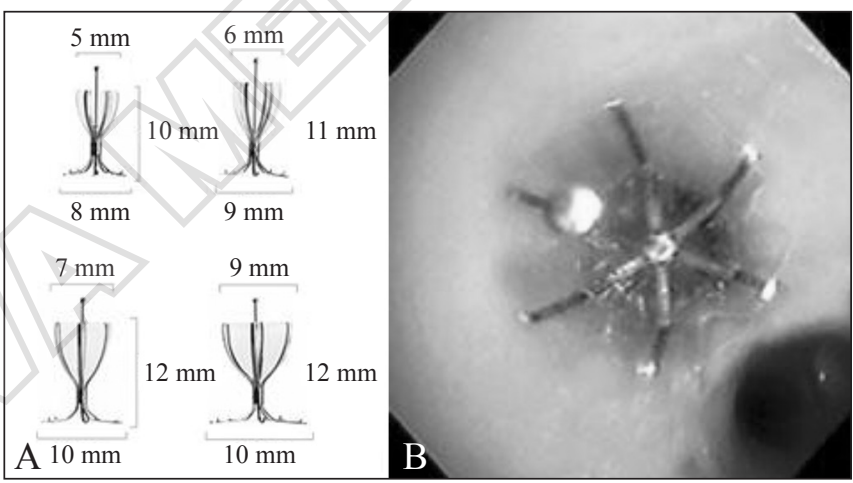

Figure 5.- Spiration IBV valve System; B) bis IBV valves.

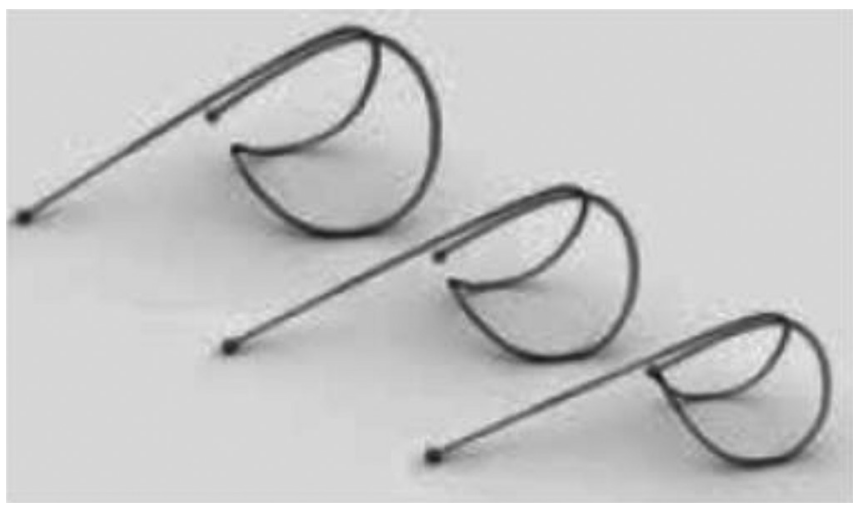

Figure 6.-Endobronchial coils. 


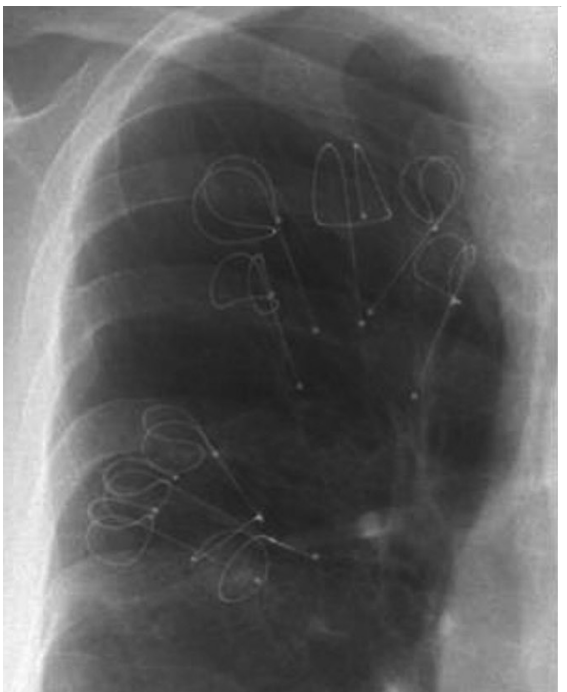

Figure 7.-Coils bilateral treatment.

the discretion of the bronchoscopist and anesthesiologist. A complete treatment includes two treatments: the first side (upper or lower lobe Coil implantation) followed by treatment of the contralateral side (Figure 7). Procedures must be staggered (contralateral procedure should be scheduled approximately 2 months after first treatment), such that both lungs are not treated during a single procedure. Lobar targeting for Coil implantation will be based on QCT densitometry analysis provided by dedicated software.

AeriSeal

The AeriSeal system is designed to function at the level of the small airways and alyeoli by blocking collateral channels and preventing gas from entering the region, causing absorption atelectasis (Figure 8).

Thus, its efficacy is not affected by collateral ventilation. The cross-linking agent used in the AeriSeal system is $4.5 \mathrm{~mL}$ of $2.1 \%$ (weight/volume) aminated polyvinyl alcohol and $0.5 \mathrm{~mL}$ of $1.25 \%$ (weight/volume) glutaraldehyde. This solution is mixed with $15 \mathrm{~mL}$ of air to produce the AeriSeal foam, which is delivered using a bronchoscope through a catheter into a pulmonary segment. Once the apposing lung parenchyma surfaces within the treated area are close enough to one another, the adhesive film seals the area closed to ensure a persistent clinical response. The most diseased areas of the lung on HRCT are treated. 15

\section{Vapor}

The InterVapor System consists of a reusable InterVapor Generator, a disposable InterVapor Catheter, and an In-
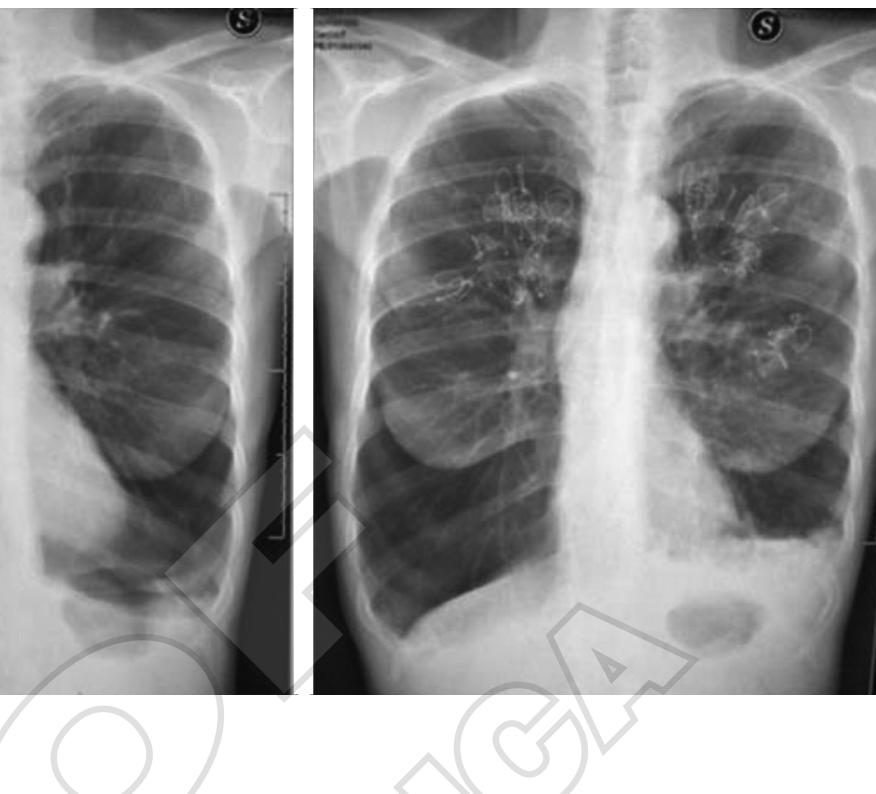

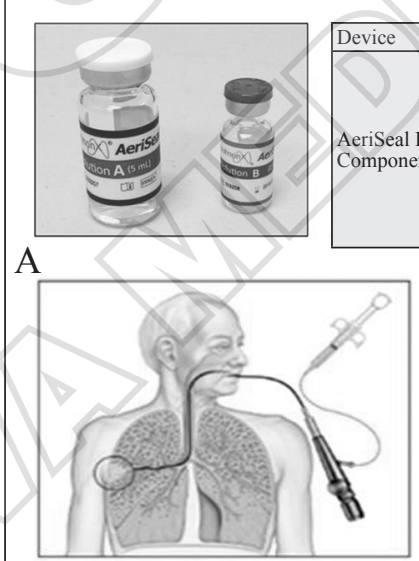

1 AeriSeal Foam Sealant administered into a pulmonary subsegment.

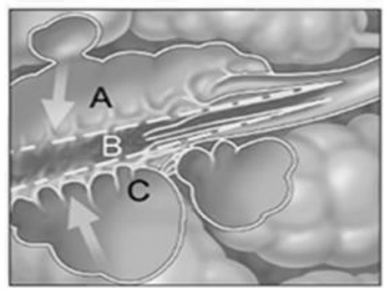

3 The treated area collapses over time B

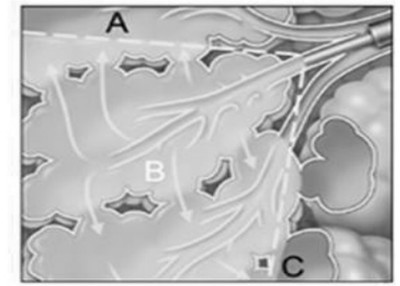

2 Close up view of sealed pulmonary subsegment.

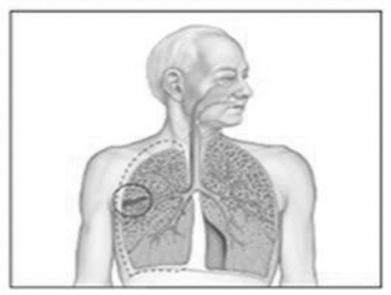

4 Lung volume is reduced as treated area collapses.
Figure 8.-A) AeriSeal components; B) bis AeriSeal treatment.

terVapor Personalized Procedure Program (IP3). The reusable InterVapor Generator provides power and is connected to a disposable InterVapor Catheter that is used to 

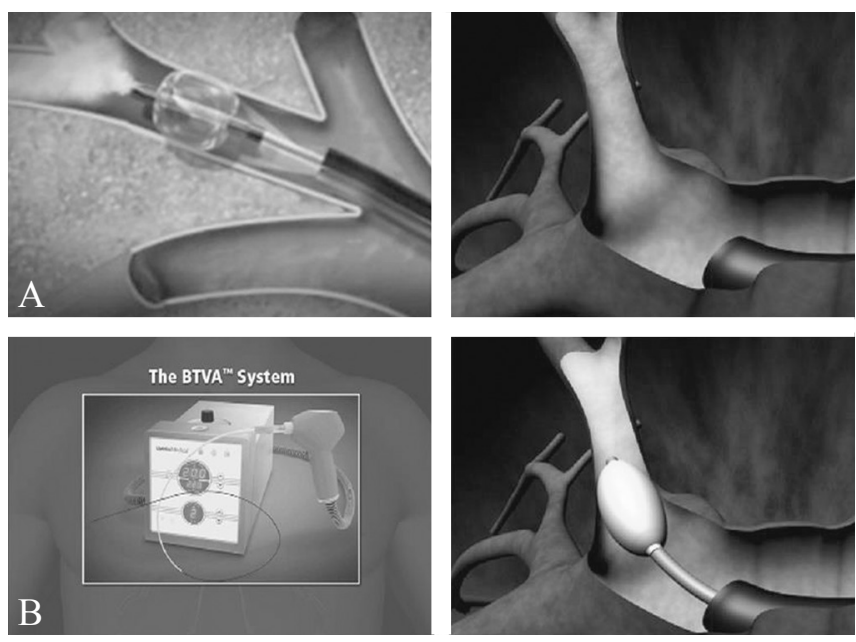

Figure 9.-Intervapor procedure; B) BTVA System.

deliver heated water vapor through a bronchoscope channel to targeted emphysematous upper lobe segments. The treatment plan (IP3) provides the duration (dose) of vapor treatment to each target airway based on the individual's specific tissue mass determined from high resolution computed tomography (HRCT) (Figure 9).

\section{Complications}

\section{Valve complication}

Pneumothorax is a possible complication following endoscopic valve therapy in emphysema patients and it occurs due to the rapid target lobe volume reduction. It may be a predictor of clinical benefit.

Although pneumothorax does not impair the clinical status in the majority of patients during follow-up, this complication needs to be considered a serious adverse event and an emergency in patients with advanced emphysema and limited respiratory reserve.

Adequate pneumothorax management, including chest tube insertion, valve removal, or surgical interventions, should be available in any institution performing endoscopic valve therapy

Optimal patient selection improves results, but also the likelihood of pneumothorax. There is, however, little evidence of what its occurrence could be influenced by.

Gompelmann et al. ${ }^{16}$ identified low attenuation volume of the ipsilateral untreated lobe, ipsilateral untreated lobe volume/hemithorax volume ratio, emphysema type, pleural adhesions and RV as significant predictors of pneumothorax. However, no single risk factor has been identified that could influence the decision making whether and how a patient should be treated or excluded from valve therapy because of a potentially fatal tension pneumothorax.

Recommendations for the treatment of a pneumothorax after valve treatment.

Other serious adverse events include COPD exacerbation, pneumonia, hemoptysis and death did not show significantly higher prevalence in valve treated patients compared to controls. However, care providers must be aware of potentially fatal complications, such as post-valvular pneumonia with septic shock or severe hemoptysis with subsequent respiratory failure. Recently, a case of severe hypersensitivity pneumonitis in a patient with Nickel allergy treated with endobronchial valves. In addition, the authors detected loss of Nickel ions from valves in in vitro experiments. Although a causative relationship remains indeed speculative and valves are merely made of Nitinol (nickel alloy) rather than nickel, valve placement in patients with distinct symptoms of Nickel allergy should only be done with caution.

\section{Coil complication}

The most common serious adverse events of BTVA treatment include COPD exacerbation (24\%) and pneumonia or pneumonitis $(18 \%)$. Therefore, we recommend vigilance to proactively detect and treat these complications. As mentioned before, each patient treated with BTVA should receive prophylactic antibiotic therapy to minimize the risk of respiratory exacerbations and bacterial superinfection. Systemic steroids may improve symptoms related to the inflammatory process. A new entity has emerged that runs any the term: "coil-associated opacities." The radiological finding is difficult to distinguish from pneumonia. The awareness has been increasing after the publications of these trials, the clinical importance is not really clear. In fact, the true pathogenesis of these opacities is unknown. In addition, it is a matter of debate, if coil-associated opacities may enhance the beneficial effect on pulmonary function on the long-term.

Though not frequent, patients who develop acute respiratory failure may require mechanical ventilation; however, non-invasive ventilation is the first treatment choice. In case of sepsis due to pneumonia, intensive treatment medicine and standard therapeutic strategies according to the guidelines are recommended.

Hemoptysis can be observed in 1-3\% of cases following BTVA treatment. It can be assumed that the hemoptysis is a consequence of the acute inflammatory reaction that is accompanied histologically by alveolar damage, bron- 
chitis, bronchiolitis, and bronchopneumonia. Antibiotic therapy and steroids may reduce the inflammatory process and thus minimize the risk of bleeding.

Pneumothorax is a rare complication following BTVA treatment. Despite this, a chest X-ray should be performed following the procedure to exclude this complication. In case of pneumothorax, treatment does not differ from the usual pneumothorax management. ${ }^{13}$

\section{Aeriseal and vapor complications}

After BLVR with vapor, the most frequent complications were lower respiratory tract infection or pneumonia in $18-25 \%$, followed by COPD exacerbation in $20-24 \%$ and self-limiting hemoptysis in $2-7 \% .{ }^{17}$ Most of the respiratory adverse events occurred in the first 90 days after treatment, and all of them were treated with standard medical care with corticosteroids and antibiotics. Only a minority of patients $(7 \%)$ in the treatment arm required significant medication intervention (intensive care unit stay or re-bronchoscopy).

After aeriseal treatment patients can develop post-treatment acute inflammatory response (PAIR) with fever, dyspnea, chest pain, cough, elevated inflammatory markers. Symptoms generally resolve within 3 days, and inflammatory markers generally normalize within 7 days.

Severity and duration may be reduced by prophylactic corticosteroids and antibiotics.

In case of PAIR patients should be assessed on day 7 to determine if they have returned to baseline dyspnea, exercise tolerance, and supplemental oxygen use. Patients who have not returned to baseline should continue a low-dose, extended corticosteroid taper for an additional 2 weeks. Symptoms of prolonged PAIR generally resolve within 2-3 weeks.

Potential severe post-treatment adverse events may include but not limited to COPD exacerbation, pneumonia and respiratory failure.

Patients may receive additional treatments in a separate treatment session.

Patients should not undergo additional treatment sessions until any post-treatment inflammatory response has resolved. 15

\section{Core basic skills}

- Competency in flexible and - optional - rigid bronchoscopy, as discussed more in detail in other contributions of this special issue (see article on "Flexible bronchoscopy and basic biopsy technique: endobronchial biopsy, transbronchial biopsy, TBNA, ROSE, BAL, brushing" and "Rigid bronchoscopy");
- active participation within the multidisciplinary team, with radiologists, pathologists, thoracic surgeons;

- sedation and oxygenation techniques, and management of related complications (see section: "Sedation in IP");

- clinical and radiographic (CT Scan and PET) evaluation of the patient, his/her comorbidities, risks and management of difficulties and complications;

- radiographic/endoscopic correlations;

- technique of preparation, loading and delivery of the different devices;

- technique for the removal of the various devices;

- prevention and management of possible complications (pneumothorax, respiratory failure, pneumonia, bleeding, exacerbation of COPD);

- management of the patient's follow-up.

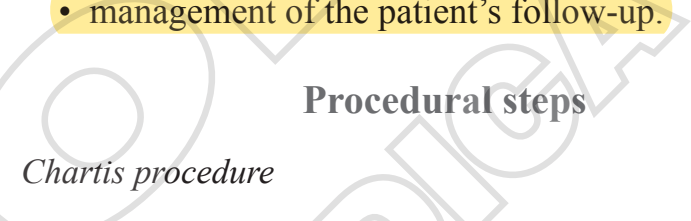

Chartis procedure is performed during a bronchoscopy. Once the patient is ready to undergo the bronchoscopy, there are a number of important nuances that can make the procedure easier. It is important that the patient is adequately medicated to provide anxiolysis, analgesia, and topical anesthesia to ensure patient comfort and minimize movement. General anesthesia is the preferred and recommended method for the Chartis measurement and for valves and coils placement due to the ease of airway and patient management.

Individual Institutional sedation guidelines should be followed. Optimal sedation during the Chartis procedure is critical, as the patient must exhibit enough tidal breathing in order to validate the detection of a CV-negative target, but must be adequately sedated to avoid coughing and/ or secretion production. A moderate sedation is ideal in prevention of respiratory failure and increased carbon dioxide retention, and deep sedation should be considered at facilities with appropriate ventilatory support and recovery resources. The choice of medications for good patient management is typically determined by institution-specific guidelines and practices.

Before starting Chartis assessment it is mandatory to:

- test the balloon by injecting approximately $1 \mathrm{~mL}$ (cc) of air and close the stopcock (Figure 10);

- inspect the balloon to ensure that it holds air volume. If the balloon holds air volume, it is ready for use (Figure 11);

- open the stopcock, retract the plunger of the syringe to remove the injected air, and close the stopcock; 


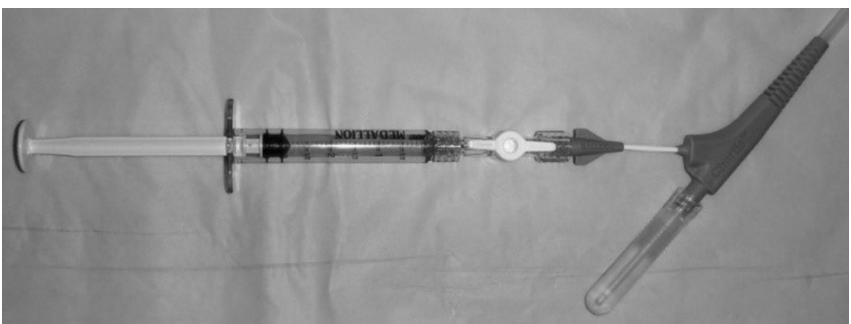

Figure 10.- Test the balloon by injecting approximately $1 \mathrm{~mL}(\mathrm{cc})$ of air and close the stopcock.

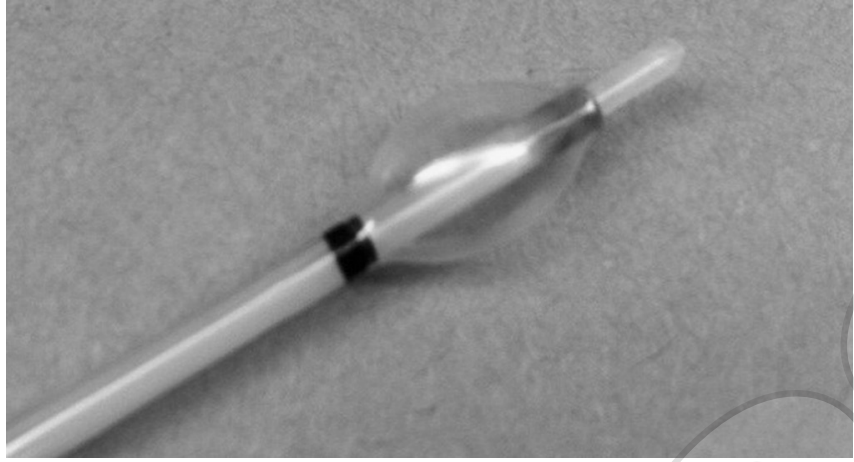

Figure 11.- Inspect the balloon to ensure that it holds air volume.

- connect the $\mathrm{AC}$ adapter to the back of the console;

- power on the console;

- unplug the 3-red quick-disconnect plugs from the sockets ( 1 on the front, 2 on the side). The plugs remain disconnected during console operation;

- step 1 - press "assess" to begin;

- step 2 - select language;

- to enter patient information, click on the PATIENT ID field. A keyboard will pop-up. In patient name fields enter initials only;

- sequentially enter information in each field. Click OK.

- Click SAVE on the patient information screen.

- Select Lobar and choose lobe for EBV assessment (Figure 12).

- Attach the connector set to the Chartis console. The connector set is included in the Chartis catheter packaging;

- lubricate the distal tip of the catheter;

- position the bronchoscope into the target airway;

- suction the target airway to clear it of mucus and fluids;

- outside of the ostium of the target lobe insert the balloon into the bronchoscope, and the black marker of the balloon catheter should be visible prior to balloon inflation. Note that the obturator should be retained within the

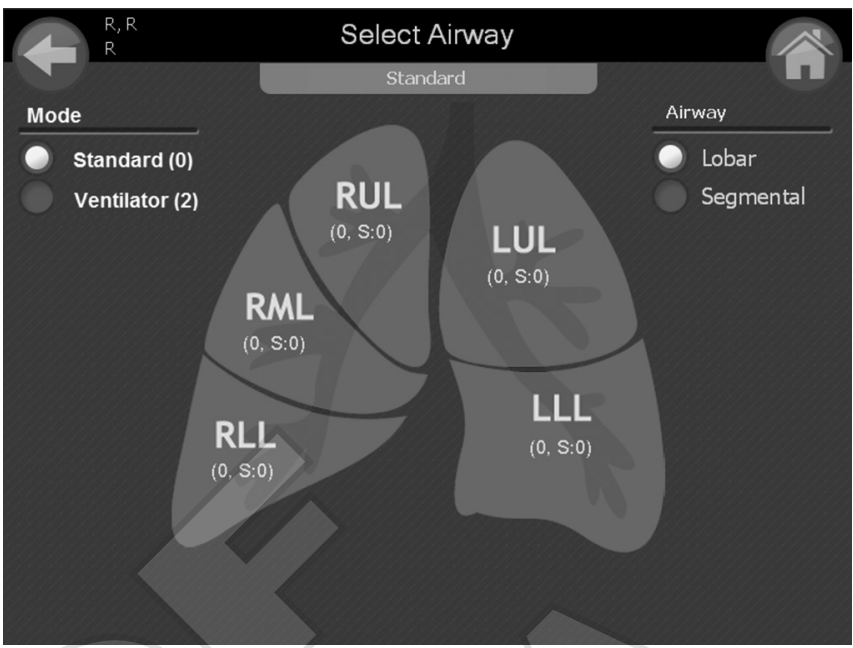

Figure 12.- Select Lobar and choose lobe for EBV assessment.

balloon catheter to prevent the catheter from kinking and prevent secretions from entering the airflow channel (Figure 13);

- position the balloon in the target airway location;

- remove the obturator from the catheter (Figure 14);

- connect one end of the three-way stopcock to the connector set;

- connect the other end to the main lumen port on the catheter (Figure 15);

- flush the catheter's main lumen:

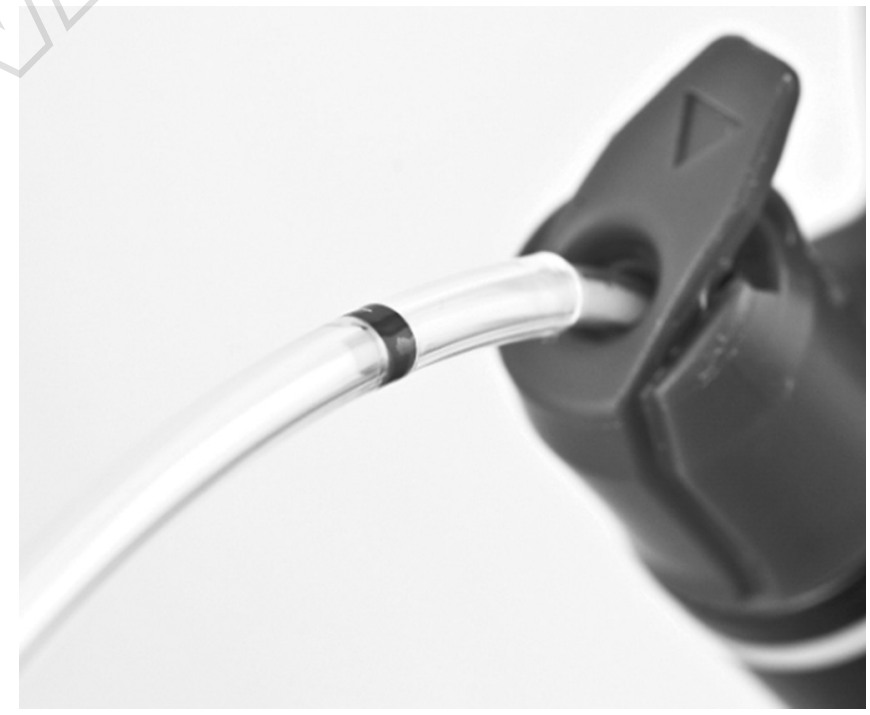

Figure 13.- The obturator should be retained within the balloon catheter to prevent the catheter from kinking and prevent secretions from entering the airflow channel. 


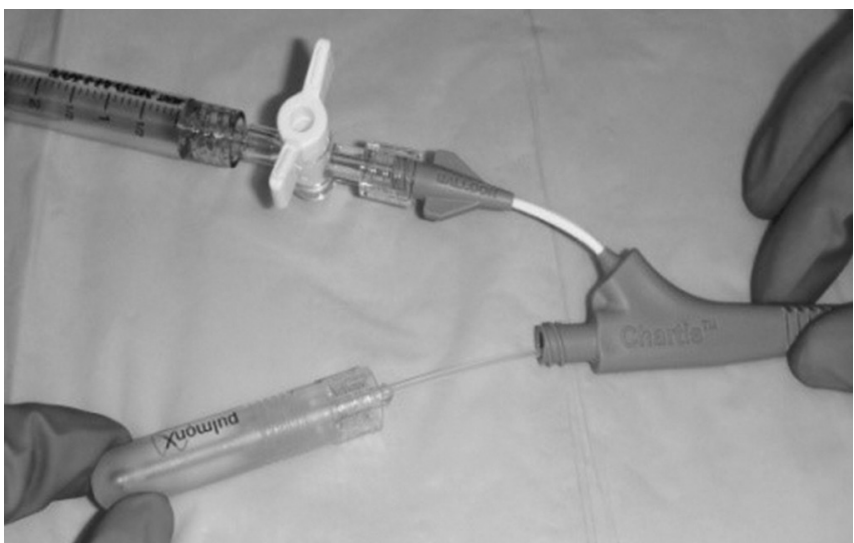

Figure 14.- Remove the obturator from the catheter.

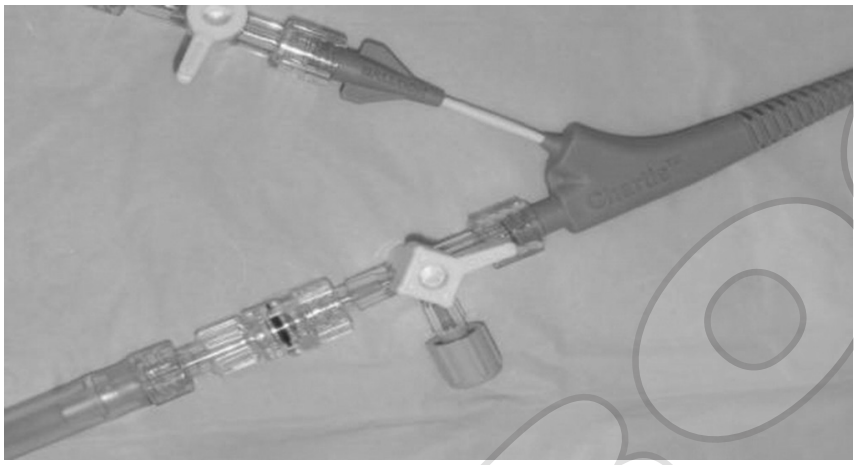

Figure 15.-Connect the other end to the main lumen port on the catheter.

- 1) connect a fully retracted 10-cc syringe;

- 2) ensure the syringe port is in the OFF position;

- 3) depress the syringe's plunger to build up pressure 1 (Figure 16);

- 4) rotate the stopcock valve away from the catheter to allow flow from the syringe to the catheter lumen;

- 5) depress the syringe to empty it of remaining air (Figure 17);

- 6) detach the syringe from the stopcock and repeat;

- 7) rotate the stopcock, keeping the main catheter lumen open to the connector set.

- 8) Inflate the catheter balloon:

- 9) Using the syringe on the BALLOON port, inject up to $3 \mathrm{~mL}$ (cc) of air as needed to create a seal in the airway under constant visualization - the light source from the scope should be reflected off of the balloon;

- 10) close the stopcock to secure the inflated balloon (Figure 18).

- 11) carefully inspect the positioning of the catheter to ensure that there is adequate contact between the bal-

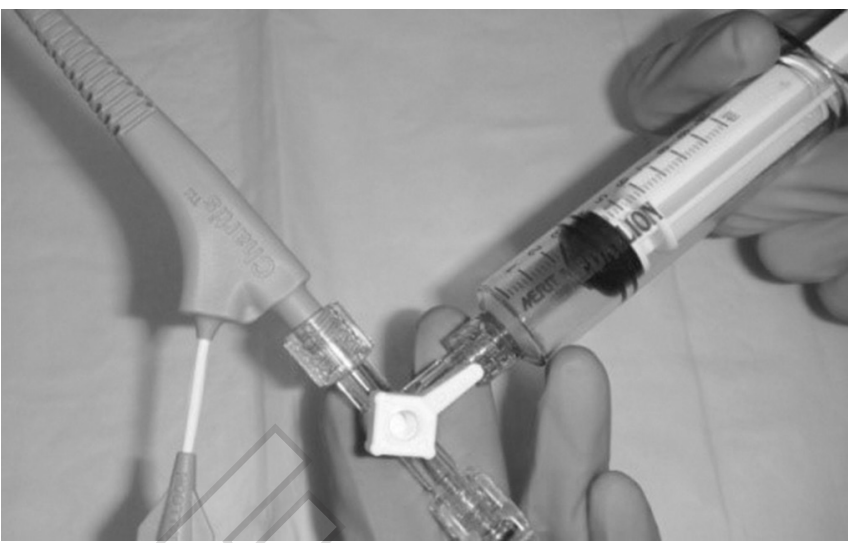

Figure 16.-Depress the syringe's plunger to build up pressure.

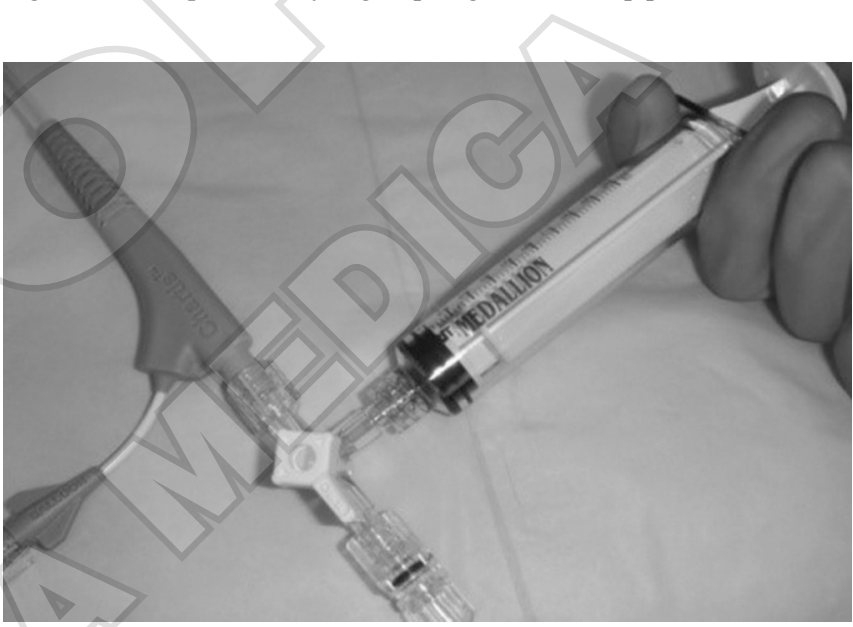

Figure 17.-Depress the syringe to empty it of remaining air.

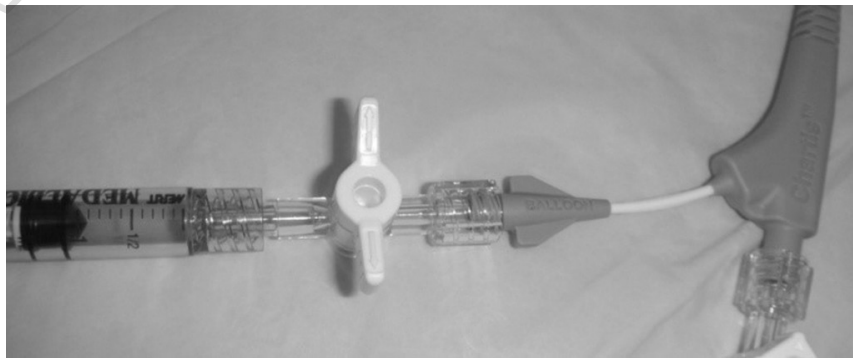

Figure 18.-Close the stopcock to secure the inflated balloon.

loon and the airway wall. Circumferential blanching of the mucosa indicates an adequate seal. Once verified, the bronchoscope should be gently advanced over the Chartis catheter and rested against the balloon to allow visualization thru the balloon and ensure that the distal end of the catheter is not being obstructed by secretions, the airway wall, or septum. 
Once the stylet is removed, regular flow should be seen on the Chartis display prior to initiating the assessment.

Perform the assessment for up to 6 minutes, assuming there is good balloon contact. Care should be taken to closely monitor for catheter obstruction, as obstruction can cause an immediate drop of the flow curve and substantially extend the measurement.

Once the flow is visualized and measured, the trend is typically quite evident. However, it can take some time to remove the volume of air from the target lobe in hyperinflated patients prior to seeing a downward trend in the flow reading.

It is recommended that the operator observes both a significant volume of air recorded $(800-1000 \mathrm{~mL})$ and a reasonable assessment time of at least 5-6 minutes to conclude a positive assessment of collateral ventilation.

The slope of the flow decrement curve depends on the compliance and pressure of the lobe being measured; however, the decrease should be steady if the lobe is CV negative.

The validity of measurement can be confirmed by continuing the measurement after stopping the assessment without deflating or moving the balloon; a notable spike in the expiratory flow once the assessment is stopped, confirms that the measurement was correct.

The assessment of collateral ventilation with Chartis

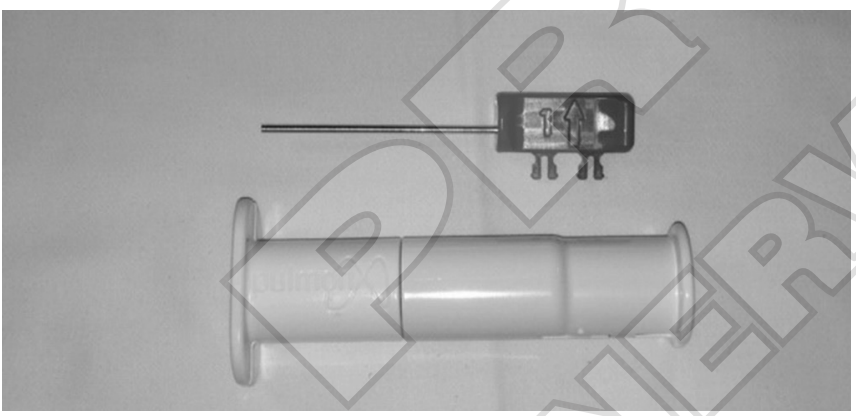

Figure 19.- - Step 1: remove pusher (labeled as 1).

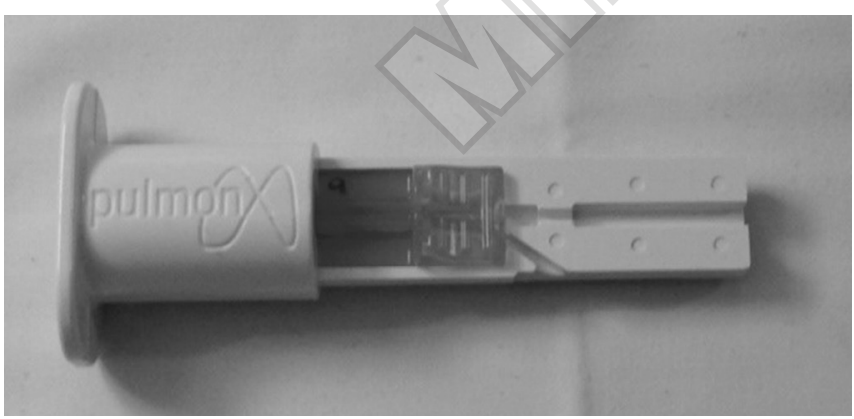

Figure 21.-Loaded components. system can be inconclusive in about $16 \%$ of the patient's CV status is confirmed by a gradual decrease and eventual cessation of expiratory airway flow in addition to a corresponding increase in resistance.

Proceed to assess a secondary target lobe if available.

BLVR with valves

\section{EBV valve}

Two different loading components are available: Blue is for 4.0 EBV and Green is 5.5 EBV.

Step 1: remove pusher (labeled as 1) (Figure 19);

step 2: pull loading components completely apart (Figure 20, 21);

step 3: pull valve compressor back (Figure 22, 23);

step 4: place delivery catheter in groove, then release valve compressor so that it slides over the distal end of the EDC;

step 5: hole to insert pusher (Figure 24);

step 6: insert pusher (Figure 25);

step 7: advance pusher with thumb;

step 8: ensure pusher completely advanced (Figure 26); step 9: retract valve compressor (Figure 27);

step 10: remove delivery catheter from groove (Figure $28)$;

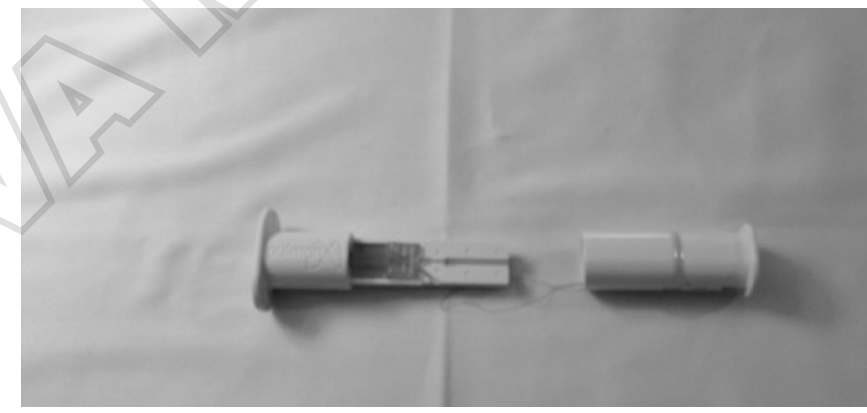

Figure 20.-Step 2: pull loading components completely apart.

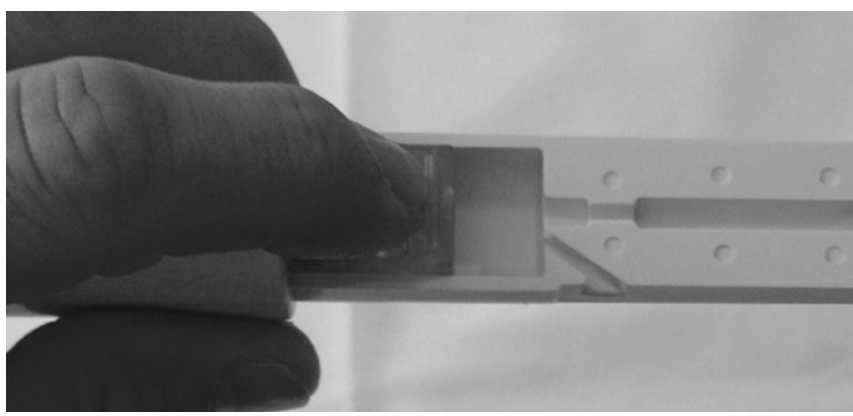

Figure 22.--Preparing valve compressor. 


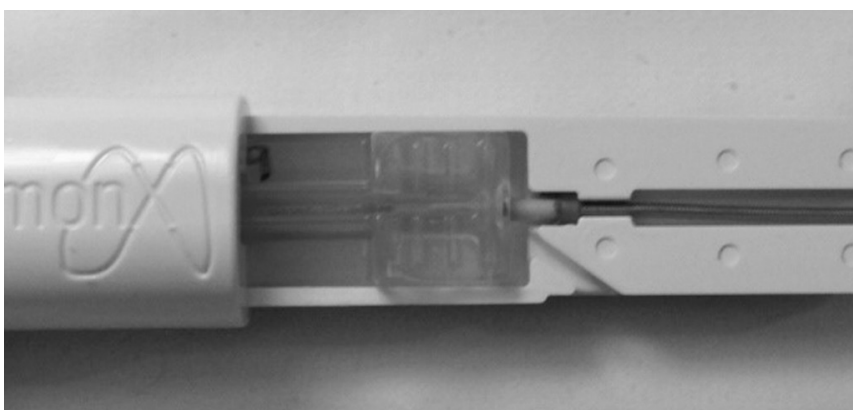

Figure 23.--Valve compressor ready.

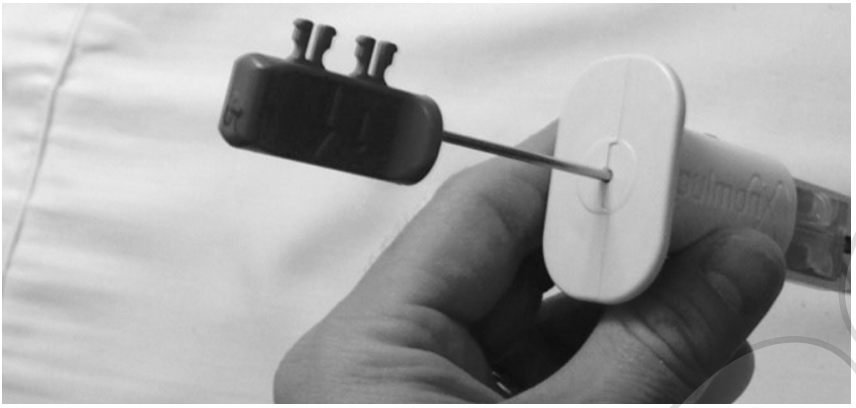

Figure 25.- - How to insert pusher.

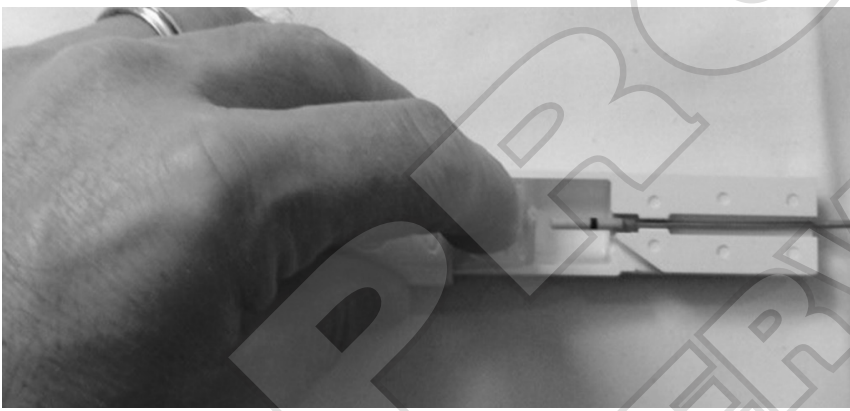

Figure 27.-_Valve compressor.

step 11: confirm valve located in delivery catheter (Figure 29).

\section{Placement}

Consideration should therefore be given to first placing the valves in the more distal and least accessible airways, prior to the easier more accessible proximal airways.

\section{Ideal positioning}

- Retention portion of valve inside lumen;

- valve and valve protector proximal to ostium;

- valve is centered within bronchus.

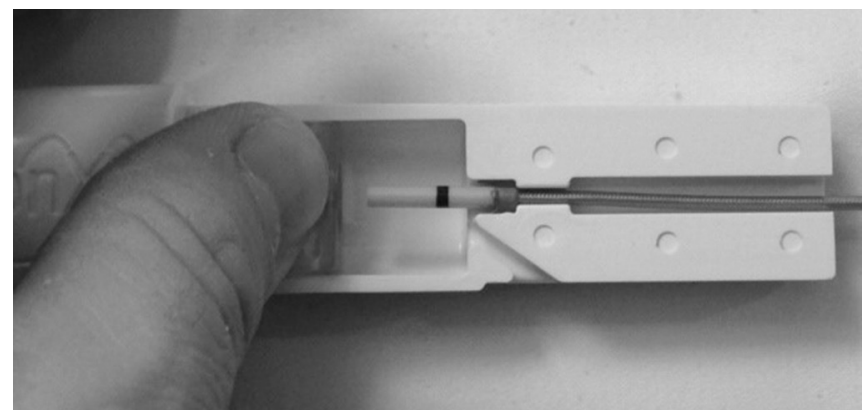

Figure 24.- - The pusher.

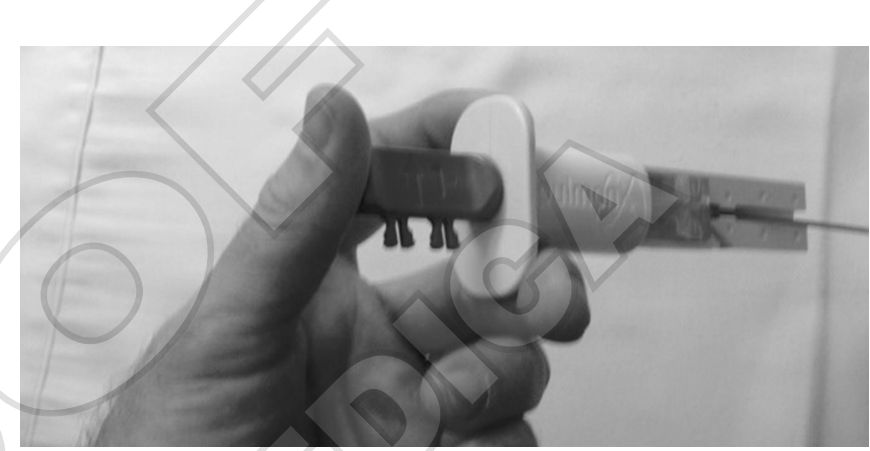

Figure 26.-Placed pusher.

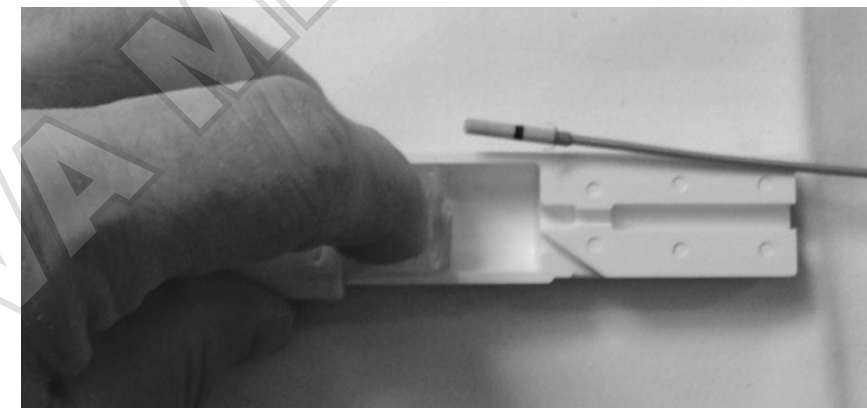

Figure 28.-Delivery catheter.

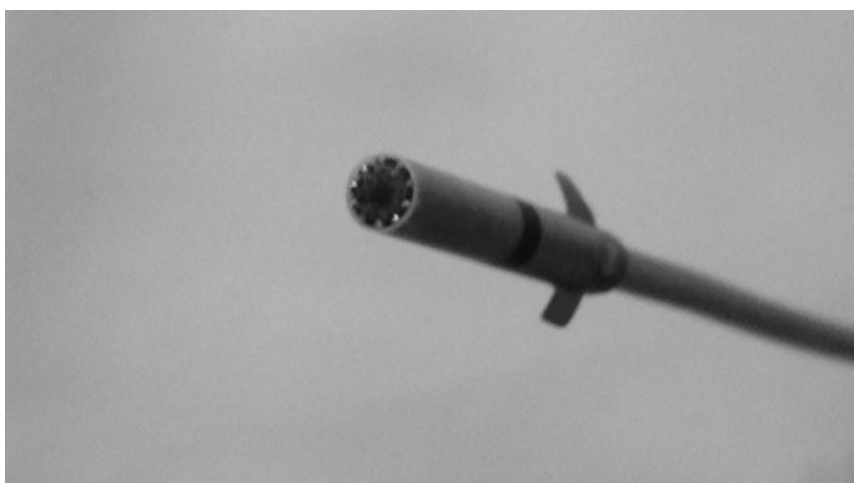

Figure 29.- Valve located in the delivery catheter. 


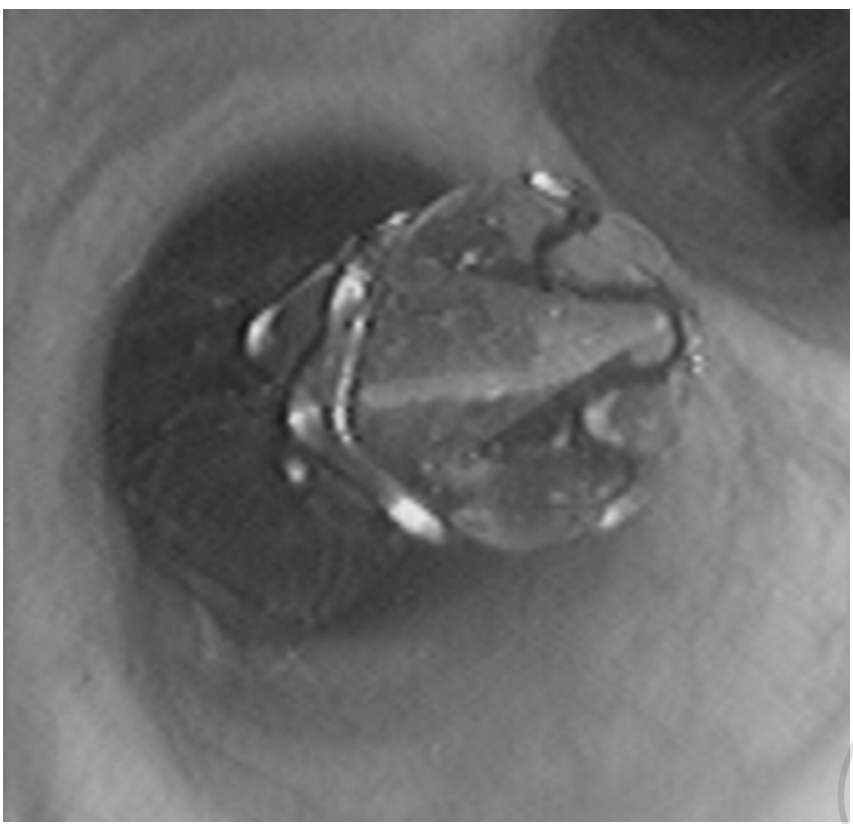

Figure 30.--Improper placement of a valve.

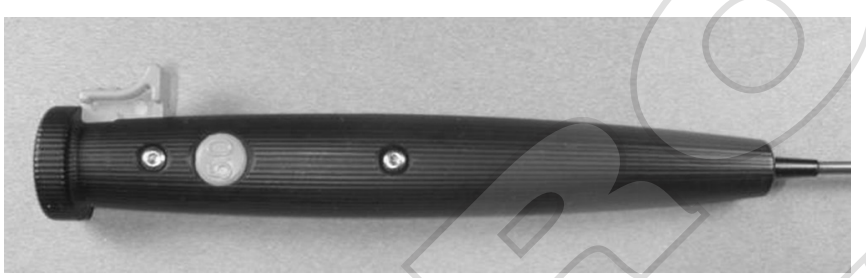

Figure 31.- Actuator in fully retracted / locked position.

Improper placement of a valve

- For proper sealing and to avoid expectoration, retention struts must be distal to ostium (not protruding);

- if too proximal, remove and replace valve;

- do not attempt to reposition the valve;

- never reuse a valve (Figure 30).

\section{EBV deployment}

- Once delivery catheter housing in proper position:

- press down and forward on the handle actuator to deploy the Zephyr EBV;

- use only moderate force to deploy the Zephyr EBV. If excessive resistance is met while deploying, stop and remove the system. Discard and load another new Zephyr valve and delivery catheter (Figure 31 ).

Press down and forward to deploy Zephyr EBV.

A valve can be positionated through two different technique:

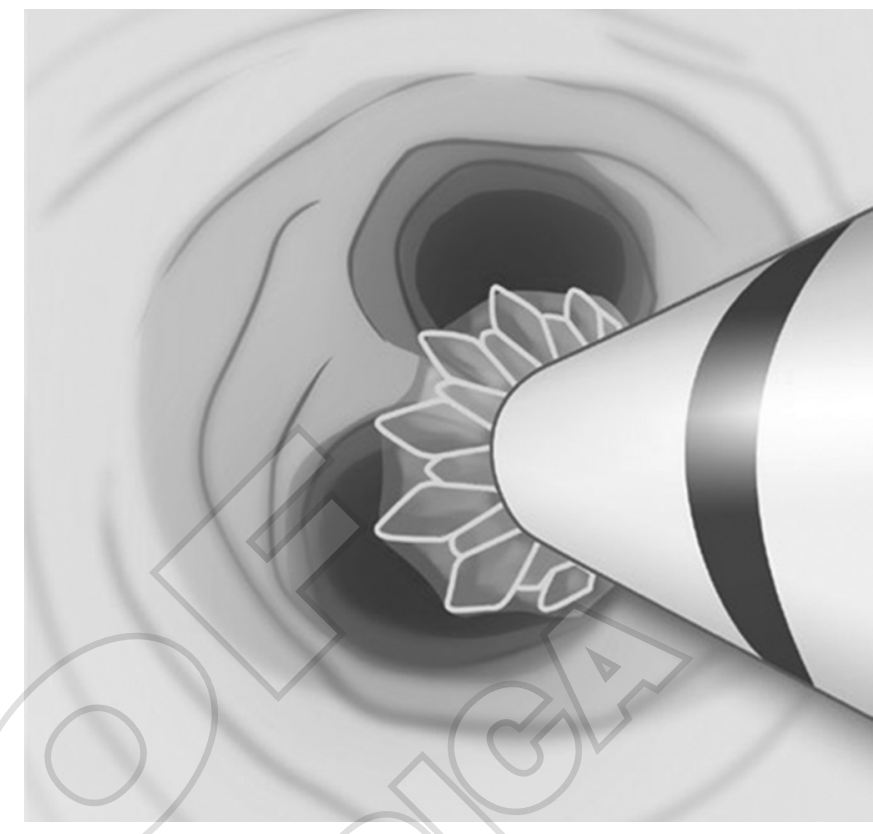

Figure 32.- Short segment technique.

Short segment technique (Figure 32):

- advance the catheter so that the marker on the tip of the catheter is visible and then slowly, partially release the valve proximal to the bifurcating carina;

- the entire unit should be then advanced to the carina and then fully deployed; this ensures the valve blocks all airways distal to the target bronchus.

Long segment technique (Figure 33):

- position the depth marker slightly distal $(1 \mathrm{~mm}$ to 2 $\mathrm{mm}$ ) to the ostium of the target segment and deploy the valve;

- it is important to note that a valve should never be deployed before visualizing the location of the distal carina to ensure the valve is correctly positioned to block all the airways of the target segment.

For proper placement, proximal end of retention strut must engage bronchial wall distal to ostium sizing requirements

There are two measurements that must be made prior to deployment of the EBV to ensure you have the correct size valve for optimal performance:

1. The physician must measure the diameter of the target lumen at the ostium to determine what size valve to use and confirm the lumen is within our size ranges: 4.0 $\mathrm{mm}$ to $8.5 \mathrm{~mm}$;

2 . the physician must measure the length from the ostium of the lumen to the distal bifurcating carina to ensure 


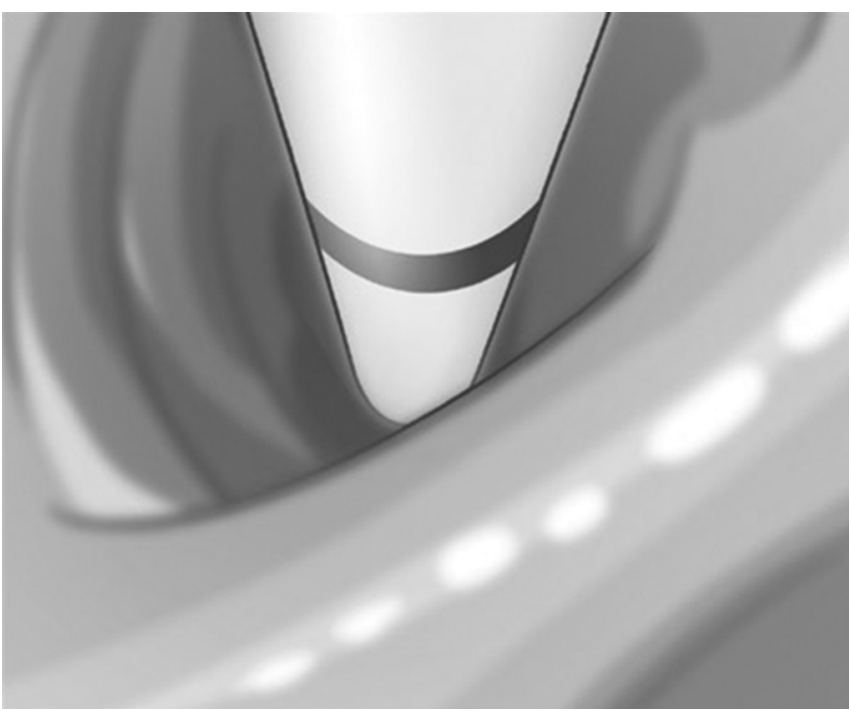

Figure 33.--Long segment technique.

there is enough length to house the retention portion of the EBV.

Using delivery catheter for required measurements:

delivery catheter allows physician to objectively measure the target segment to ensure:

1. correct diameter valve is used;

2. appropriate length between the ostium and bifurcating carina to place a valve.

The wings on the catheter should be used to identify the minimum and maximum diameter of the valve (Figure 34). The longer wings, representing the maximum diameter of the valve, should touch the airway walls at the widest point of the lumen. This can be accomplished by rotating the catheter by turning the handle when the catheter is in position. The two shorter wings indicate the smallest size of the bronchial segment that can be treated. When in doubt, physicians should oversize the diameter of the valve if they have the airway depth to do so, as this will provide a tighter seal.

Retention portion of EBV needs to be in lumen of target.

The depth marker on the endobronchial delivery catheter can be used to help determine the distance that the valve will protrude from the landing zone and validate the optimal order of placement. It is very important to ensure the target segment has enough length between the distal bifurcating carina and ostium to land the body of the valve. This can be accomplished by ensuring the length of the airway segment is greater than the distance from the tip of the delivery catheter to the blue marker (Figure 35). This distance represents the length of the valve housing

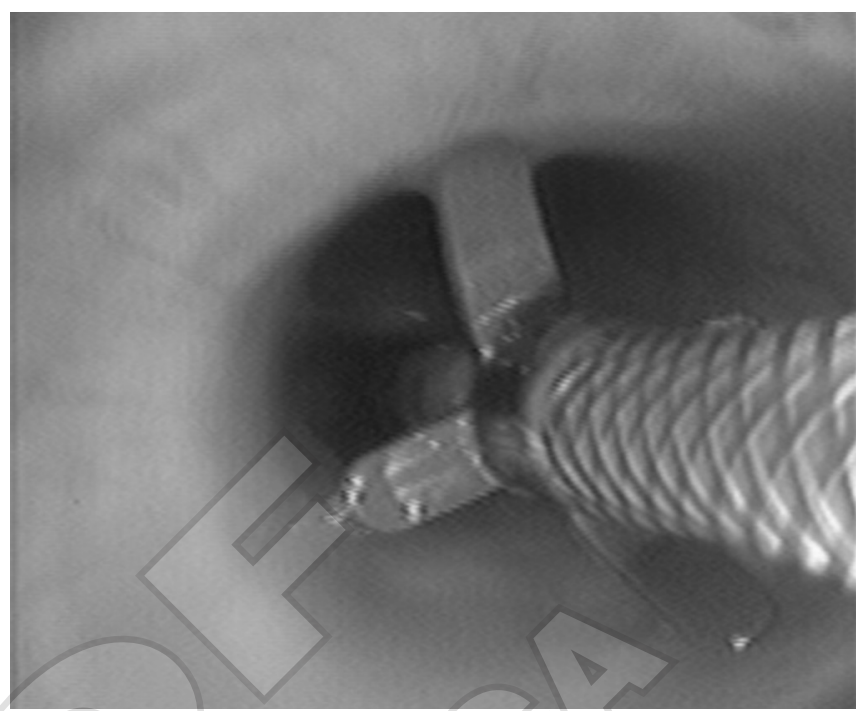

Figure 34.-Wings of the catheter.

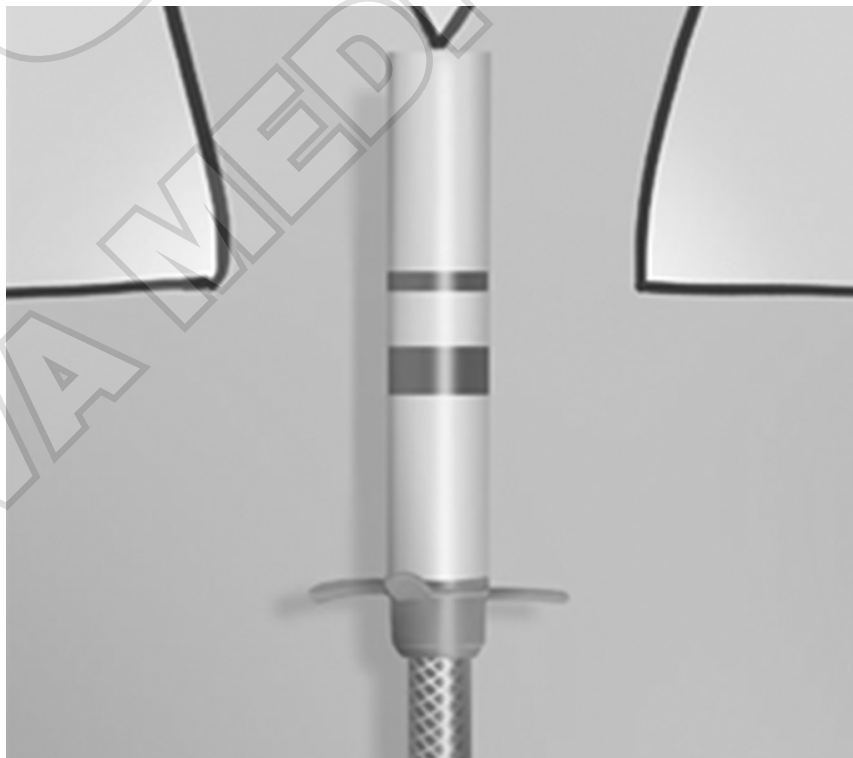

Figure 35.--Length of the airway segment.

that should be seated behind the ostium to ensure correct placement.

Warning: do not place the Zephyr EBV such that the distal end of the retainer is placed beyond the distal carina of the target bronchus (Figure 36).

- This leaves one branch untreated and will result in a leak and lack of volume reduction;

- this may also result in proximal migration of the implanted Zephyr EBV; 
Figure 36.-Distal end of the retainer beyond the distal carina.
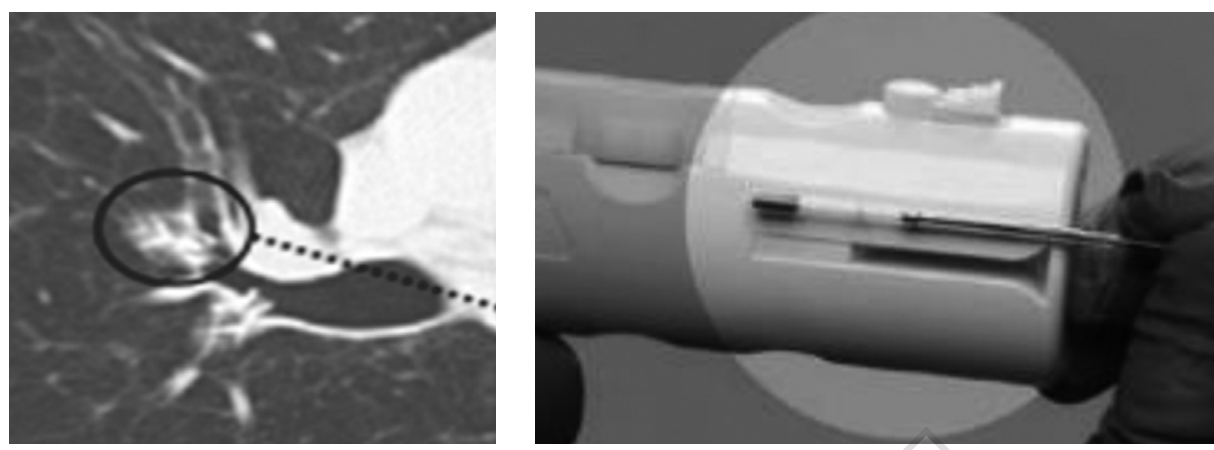

Figure 37.--Loading the spiration valve.
- precaution: attempting to reposition the Zephyr EBV by grasping the valve protector portion of the retainer may result in device damage.

During the placement of a valve, it can be helpful to locate the tip of the delivery catheter just outside of the bronchoscope and then maneuver as close as possible to the target area. After valve sizing measurements are made, advance the catheter so that the marker on the tip of the catheter is visible and then slowly, partially release the valve proximal to the bifurcating carina.

The "low profile" EBV-TS4.0-LP valve is often useful for placement in short segments.

\section{IBV valve}

Prior to the procedure, a balloon is calibrated to size the airways. The calibrated balloon is used to determine the appropriate valve size for placement (Figure 37).

1) remove green plunger clip;

2) depress plunger;

3) rotate yellow lock;

4) lift and pull catheter from loading tray.

1. Carefully insert the catheter into the working channel of the bronchoscope. Only use a bronchoscope with a 2.6 $\mathrm{mm}$ working channel or larger. Do not bend, kink or Jam the distal end of the catheter while inserting. A kink may prevent the valve from deploying from the catheter. If this occurs, discard the catheter and valve.

2. While the bronchoscope is in a relaxed position, advance the catheter until the stabilization rod and removal rod tip are visible.

3. Remove the red safety clip from the deployment handle. While looking at the removal rod tip through the catheter, slowly depress the white deployment handle to eliminate any gap between the removal rod tip and the stabilization rod (S). Retract the catheter until the end of the catheter tip is just visible at the end of the bronchoscope and does not interfere with its operation.
4. Under bronchoscopic observation, advance the bronchoscope to the deployment location.

5. Position the bronchoscope so that the target airway location is visible and the tip of the catheter can be directed into the target site without bending or kinking the catheter.

6. Advance the catheter to the target location for valve deployment.

7. Position the catheter so that the VDG or the proximal tips of the membrane struts (S) are visible and align the VDG/struts with the target location in the airway. The valve may settle $1-2 \mathrm{~mm}$ distal over time.

8. Important: Hold the catheter in place at the opening of the insertion port of the bronchoscope.

9. Under bronchoscopic observation, depress the white deployment handle, which retracts the catheter sheath and releases the valve.

10. Once the valve is completely deployed, remove the catheter from the bronchoscope.

11. Examine the valve for opening, position, and fit. The valve should be opened and opposing against all borders of the airway.

1. Removal of valves should be conducted under bronchoscopic observation. It is recommended that valves should be removed through an endotracheal (ET) tube.

2. Insert the appropriate forceps through the working channel of the bronchoscope, directing the forceps to the target location (Figure 38).

3. Grasp the removal rod with the appropriate forceps and gently pull the valve until it is dislodged from the airway wall. Use care to make sure that the removal rod does not get caught in the fenestration of the forceps when removing the valve.

4. Before removing the valve from the trachea, pull the valve as close as possible to the end of the bronchoscope.

5. While still firmly holding onto the valve with the forceps, simultaneously remove the bronchoscope and the forceps from the patient. 


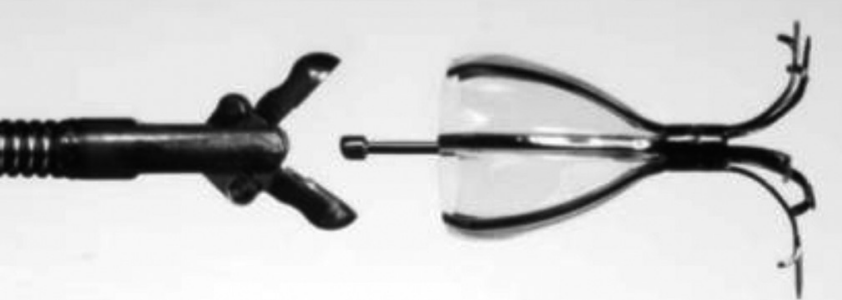

Figure 38.- Spiration valve removal with forceps.

\section{BLVR with coils step by step}

Endobronchial coils are non-blocking, shape-retaining nitinol devices delivered bronchoscopically into the subsegmental airways to induce parenchymal compression and enhance lung recoil. 10-12 coils (size 100-150 mm) are delivered in the most diseased lobes using fluoroscopy to control positioning. One lobe is treated per procedure, and a contralateral lobe is treated 1-4 months later. The proposed mechanism of action of the endobronchial coils, which act independently of collateral flow, is a combination of the physiological effects of lung volume reduction and restoration of the elastic properties of the lung tissue.

1. Navigate the bronchoscope to the target airway and position at the ostium of a segmental airway. 2. Insert both the catheter and guidewire into the working channel of the bronchoscope.

3. Advance and navigate the guidewire into the distal targeted airway under fluoroscopy guidance (Figure 39).

4. Estimate position of pleural edge and length of the airway with the guidewire and then retract the guidewire so that it is either far enough from the pleural edge (25-40 $\mathrm{mm}$ ) or a maximum of 1.25-1.5 fluoroscopy markers distal from the tip of the bronchoscope (to accommodate a 125 $\mathrm{mm}$ coil).

5. Hold the guidewire position fixed relative to the bronchoscope and advance the catheter distally but not beyond the tip of the guidewire.

6. Once the catheter is in the desired position, withdraw the guidewire tip until it is aligned with the tip of the catheter.

7. Use the radiopaque markers on the guidewire to measure the airway length.

8. Remove the guidewire from the catheter while maintaining the catheter position.

9. Select the appropriate Coil size by counting the number of radiopaque markers on the guidewire visible outside the bronchoscope. The markers indicate the minimum recommended coil size to be used. Do not count the Guidewire tip as a marker for Coil selection. The desired size

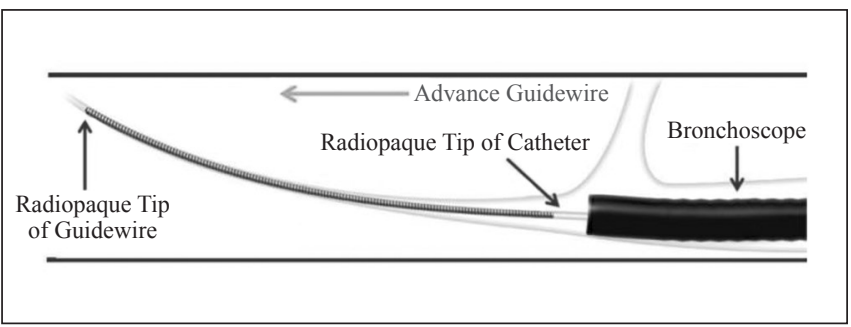

Figure 39.-Advancing guidewire into targeted airways.

coil can be loaded into the cartridge (1 radio-opaque marker or fractionally less $=100 \mathrm{~mm}$, at least more than $1.25-1.5$ of the distance between the first marker or more $=125 \mathrm{~mm}$ and if more than 2 markers consider using $150-\mathrm{mm}$ coil) (Figure 40).

10. Connect the cartridge to the Luer-lock hub of the catheter and lock into place.

11. Deliver the coil into the catheter by advancing the forceps and coil.

12. Align the distal end of the coil with the distal end of the catheter.

13. Position the coil using fluoroscopy.

14. Have an assistant hold the bronchoscope fixed relative to the patient.

15. Deploy the coil using fluoroscopy by withdrawing the catheter with one hand, while holding the coil position fixed with the forceps using the other hand.

16. Verify the position of the coil under fluoroscopy and release the coil by unlocking the forceps under gentle traction.

17. Remove the forceps from the catheter.

18. The catheter may continue to be used to repeat steps 2-17 to deploy additional coils. The coil can be removed or repositioned by reversing this implantation process.

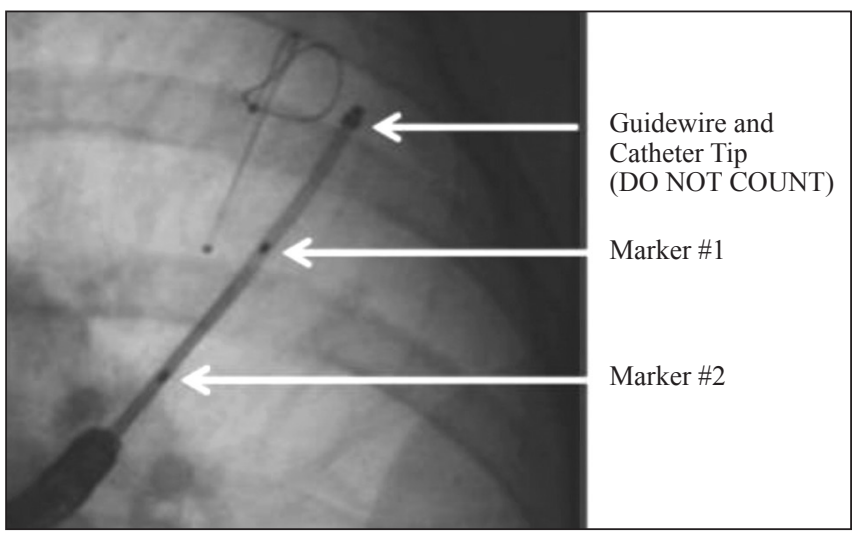

Figure 40.-Guidewire radiopaque marker band under fluoroscopy. 


\section{BLVR with AeriSeal step by step}

The AeriSeal System is intended to reduce lung volume in order to improve lung function and quality of life in patients with advanced emphysema. It functions by physically occluding both small airways and collateral air channels, causing the treated area to collapse via absorption atelectasis. It is a single use device intended to be used by pulmonologists and thoracic surgeons in a bronchoscopy suite or operating room.

First is the identification of the most diseased segments of the target lobe(s).

Then it is important to identify one subsegment within each chosen segment for treatment and a backup sites in case subsegments cannot be treated.

Treatment site selection:

- do not treat regions with pre-existing radiologic abnormalities that require follow-up;

- do not treat subsegments that are scarred or have granulation tissue due to prior pathology or valve therapy;

- no more than two lobes should be treated during a single treatment;

- no more than 4 subsegments should be targeted for treatment in a single treatment session;

- no more than 2 subsegments should be treated within a single lobe in a single treatment session;

- treating adjacent lung regions can be associated with increased incidence and/or severity of post-treatment inflammatory responses and COPD exacerbations;

- treat only one subsegment within a segment.

\section{Patient preparation}

- Follow institutional guidelines for preparing patient for bronchoscopy and anesthesia;

- pre-treat patient with short acting bronchodilator 1520 minutes prior to procedure to reduce bronchospasm;

- provide adequate anesthesia (general anesthesia or conscious sedation) to ensure patient comfort and safe airway access;

- apply topical anesthesia to minimize coughing.

Starting on the day of treatment, all patients must be administered the following prophylactic therapy:

- corticosteroids (7 days);

- antibiotics (7 days);

- NSAIDs (3 days);

- antiacids (7 days).

First doses of corticosteroids and antibiotics should be administered at least 2 hours prior to procedure.

\section{Balloon catheter preparation}

- Lubricate the distal tip of the balloon catheter with a viscous sterile lubricant for ease in passing the catheter through the working channel of the bronchoscope;

- attach the 1-way Stopcock to balloon inflation port of the catheter;

- depress plunger of sterile $3 \mathrm{~mL}$ syringe and attach to opposite end of the 1-way Stopcock. Ensure the 1-way Stopcock is in open position;

- apply a vacuum to the balloon lumen by retracting the plunger of the inflation syringe and closing the 1-way Stopcock prior to inserting the catheter into the bronchoscope;

- fill a $30 \mathrm{~mL}$ syringe with $15 \mathrm{~mL}$ of air and set aside;

- push air out of the $20 \mathrm{~mL}$ syringe and connect to Stopcock;

- position "OFF" handle of the Stopcock to point opposite the $20 \mathrm{~mL}$ syringe;

- Using a new $20 \mathrm{G}$ needle and a $6 \mathrm{~mL}$ syringe, draw up $4.5 \mathrm{~mL}$ of Solution A; remove the white cap from the middle port of the 3-way Stopcock and connect the $6 \mathrm{~mL}$ syringe containing Solution A to the port;

- confirm that the Stopcock is positioned so that the "OFF" handle points opposite the $20 \mathrm{~mL}$ syringe to prevent accidental spillage of treatment material. Deliver all of Solution A to the $20 \mathrm{~mL}$ syringe. Remove the empty $6 \mathrm{~mL}$ syringe;

- using a new $20 \mathrm{G}$ needle and a $1 \mathrm{~mL}$ syringe, draw up $0.5 \mathrm{~mL}$ of Solution B;

- select a standard flexible bronchoscope with 4.5-5.4 $\mathrm{mm}$ OD and working channel diameter $\geq 2.0 \mathrm{~mm}$ or $\geq 2.8$ $\mathrm{mm}$ for delivery with balloon catheter;

- survey the airways. Discontinue treatment if patient is not tolerating bronchoscopy or if purulence, bleeding, or an unexpected endobronchial lesion is detected;

- guide the flexible bronchoscope to the subsegment that has been selected for treatment;

- insert the balloon catheter with the obturator into the working channel of the bronchoscope;

- advance balloon catheter approximately $2 \mathrm{~cm}$ beyond the distal tip of the bronchoscope until the catheters distal balloon tip is visualized and the strain relief is engaged in the bronchoscope valve. Guide the bronchoscope to the subsegment that has been selected for treatment and position the distal tip of the balloon into the target airway;

- remove the obturator from the catheter;

- use the $3 \mathrm{~mL}$ syringe inflate the balloon as needed to create a seal in the airway. Close the one way stopcock 
to ensure balloon remains inflated. Confirm via visual inspection that the target airway is occluded. Air bubbles at the balloon should not be visible during respiration. Catheter tip should not be contacting airway wall;

- connect the $1 \mathrm{~mL}$ syringe containing Solution B to the 3-way Stopcock at the middle port. Deliver Solution B from the $1 \mathrm{ml}$ syringe to the $20 \mathrm{~mL}$ syringe that contains Solution A. This initiates polymerization.

All subsequent steps should be performed quickly to prevent the material from polymerizing before delivery.

- Remove the empty $1 \mathrm{~mL}$ syringe and connect the 30 $\mathrm{mL}$ syringe containing $15 \mathrm{~mL}$ of air to the Stopcock at the middle port;

- generate a uniform white foam by alternately pushing the plungers of the $20 \mathrm{~mL}$ and $30 \mathrm{~mL}$ syringes so that the mixture passes through the Stopcock a total of 10 cycles, therefore each plunger will need to be pushed 10 times. Two passes through the Stopcock make up one cycle. This should take between 7 and 15 seconds;

- following foam generation, ensure that the desired volume of Foam is contained in the $20 \mathrm{~mL}$ syringe. Transfer any excess Foam to the $30 \mathrm{~mL}$ syringe;

- inject the desired volume of Foam into the Catheter applying steady firm pressure to the plunger of the $20 \mathrm{~mL}$ syringe. Complete injection of the Foam should take between 5 and 30 seconds;

- keep the plunger of the $20 \mathrm{~mL}$ syringe depressed for at least 5 seconds after completion of injection to ensure that all of the Foam is delivered;

- attach a $60 \mathrm{~mL}$ syringe to the balloon catheter;

- over 5 seconds inject up to $30 \mathrm{~mL}$ of air through the balloon catheter with the $60 \mathrm{~mL}$ syringe to promote distal distribution of the Foam;

- keep the balloon catheter inflated and in position for at least 1 minute to prevent spillage of material;

- deflate the balloon catheter and remove from the bronchoscope.

\section{Treatment completion}

After all target sites have been treated, briefly inspect the large airways. Spillage of small amounts of Foam Sealant can be suctioned out through the bronchoscope. The bronchoscope should be cleaned after each patient use in accordance with the manufacturer's instructions. No special cleaning techniques are required.

- monitoring should be continued until the patient is awake and responsive;

- observe patient at least overnight in the hospital;

- discharge patient when clinically stable;
- patients should complete the full steroid + antibiotic prophylaxis regimen.

\section{Bronchoscopic thermal vapor ablation Step by Step}

For the treatment, an InterVapor catheter is guided through the airways via a standard therapeutic bronchoscope (minimum 2.8-mm channel diameter) to the diseased segment and a precise, patient specific, dose of vapor is delivered. This painless, vapor dose is delivered in 3 to 10 seconds, ablating the targeted portion of diseased tissue causing a natural, gradual reduction occurring over a 4-6-week reparative process. The amount of energy delivered is calculated based on the mass of the emphysematous segment to be ablated and is presented as calories/gram (cal/g) of diseased tissue. The vapor disperses within the emphysematous segment resulting in ablation of the segment's parenchyma and airways. Immediate cell death occurs to the diseased alveoli, bronchioles and microvascular of the segment. The body's natural healing mechanisms (e.g. macrophages, neutrophils) remove the ablated cells and results is a reduction in the mass and volume of the diseased segment. The surrounding, better functioning, segments of upper lobe as well as the middle and lower lobes expand. The healing process typically matures within the first 6 weeks following vapor ablation and is typically followed by improvements in pulmonary function and quality of life.

The InterVapor therapy typically consists of one to two procedures of Vapor treatment separated by at least three months to allow for complete healing prior to subsequent procedures.

\section{Theoretical and practical training}

BLVR is a highly complex procedure: a first-level competence is a pre-requisite for admission to training. 18

Trainees must possess in-depth knowledge of anatomy and be capable of interpreting imaging findings, especially in terms of evaluating fissures, pulmonary density by means of HRCT. In addition, trainees must be able to use and interpretate quantitative analysis of CT scan with different software.

The practical training must be based on:

- live session and clinical case discussion with emphasis on the strategy to follow in different situations;

- task trainer simulation with plastic model;

- non-technical skill simulation for the training on how to manage complications within a multidisciplinary team with anesthetist and nurses; 
- training on animal models and cadaver;

- training on patient under supervision;

In order to complete user training, each user must complete for each device the "hands-on" device training. ${ }^{19}$

\section{Quantitative assessment with logbook (minimum volume)}

No. of procedures: unknown.

\section{Qualitative assessment: mcq}

- MCQ

- case-based questionnaires;

- dops (not specific).

Outcome assessment: assessment of outcomes (mortality, survival, complications, pulmonary function, quality of life, dyspnea scores) in National and international registers (e.g. coils).

\section{References}

1. Pistolesi M, Camiciottoli G, Paoletti M, Marmai C, Lavorini F, Meoni $\mathrm{E}$, et al. Identification of a predominant COPD phenotype in clinical practice. Respir Med 2008;102:367-76.

2. Russi EW, Stammberger U, Weder W. Lung volume reduction surgery for emphysema. Eur Respir J 1997;10:208-18.

3. Criner GJ, Sue R, Wright S, Dransfield M, Rivas-Perez H, Wiese T, et al.; LIBERATE Study Group. A Multicenter Randomized Controlled Trial of Zephyr Endobronchial Valve Treatment in Heterogeneous Emphysema (LIBERATE). Am J Respir Crit Care Med 2018;198:1151-64.

4. Criner GJ, Cordova F, Sternberg AL, Martinez FJ. The National Emphysema Treatment Trial (NETT) Part II: lessons learned about lung volume reduction surgery. Am J Respir Crit Care Med 2011;184:881-93.

5. Herth FJ, Gompelmann D, Ernst A, Eberhardt R. Endoscopic lung volume reduction. Respiration 2010;79:5-13.

6. Gompelmann D, Shah PL, Valipour A, Herth FJ. Bronchoscopic Thermal Vapor Ablation: Best Practice Recommendations from an Expert Panel on Endoscopic Lung Volume Reduction. Respiration 2018;95:392-400.
7. Gasparini S, Zuccatosta L, Bonifazi M, Bolliger CT. Bronchoscopic treatment of emphysema: state of the art. Respiration 2012;84:250-63.

8. Slebos DJ, Ten Hacken NH, Hetzel M, Herth FJ, Shah PL. Endobronchial Coils for Endoscopic Lung Volume Reduction: Best Practice Recommendations from an Expert Panel. Respiration 2018;96:1-11.

9. Schuhmann M, Raffy P, Yin Y, Gompelmann D, Oguz I, Eberhardt $\mathrm{R}$, et al. Computed tomography predictors of response to endobronchial valve lung reduction treatment. Comparison with Chartis. Am J Respir Crit Care Med 2015;191:767-74.

10. Koster TD, van Rikxoort EM, Huebner RH, Doellinger F, Klooster K, Charbonnier JP, et al. Predicting lung volume reduction after endobronchial valve therapy is maximized using a combination of diagnostic tools. Respiration 2016;92:150-7.

11. Kemp SV, Slebos DJ, Kirk A, Kornaszewska M, Carron K, Ek L, et al.; TRANSFORM Study Team. A Multicenter Randomized Controlled Trial of Zephyr Endobronchial Valve Treatment in Heterogeneous Emphysema (TRANSFORM). Am J Respir Crit Care Med 2017;196:1535-43.

12. Valipour A, Slebos DJ, Herth F, Darwiche K, Wagner M, Ficker JH, et al.; IMPACT Study Team. Endobronchial valve therapy in patients with homogeneous emphysema. Results from the IMPACT study. Am J Respir Crit Care Med 2016;194:1073-82.

13. Slebos DJ, Hartman JE, Klooster K, Blaas S, Deslee G, Gesierich W, et al. Bronchoscopic Coil Treatment for patients with severe emphysema: A meta-analysis. Respiration 2015;90:136-45.

14. Gompelmann D, Eberhardt R, Herth FJ. Technology update: bronchoscopic thermal vapor ablation for managing severe emphysema. Med Devices (Auckl) 2014;7:335-41.

15. Herth FJ, Gompelmann D, Stanzel F, Bonnet R, Behr J, Schmidt B, et al. Treatment of advanced emphysema with emphysematous lung sealant (AeriSeal $\mathbb{R}$ ). Respiration 2011;82:36-45.

16. Gompelmann D, Benjamin N, Kontogianni K, Herth F, Heussel CP, Hoffmann $\mathrm{H}$, et al. Clinical and radiological outcome following pneumothorax after endoscopic lung volume reduction with valves. Int J Chron Obstruct Pulmon Dis 2016;11:3093-9.

17. Herth FJ, Valipour A, Shah PL, Eberhardt R, Grah C, Egan J, et al. Segmental volume reduction using thermal vapour ablation in patients with severe emphysema: 6-month results of the multicentre, parallelgroup, open-label, randomised controlled STEP-UP trial. Lancet Respir Med 2016;4:185-93.

18. Mullon JJ, Burkart KM, Silvestri G, Hogarth DK, Almeida F, Berkowitz D, et al. Interventional Pulmonology Fellowship Accreditation Standards: Executive Summary of the Multisociety Interventional Pulmonology Fellowship Accreditation Committee. Chest 2017;151:1114-21.

19. Corbetta L, Patelli M. Executive Summary of Training and Competence Standards for the Interventional Pulmonology Master Program in Italy. J Bronchology Interv Pulmonol 2018;25:6-8.

Conflicts of interest.-The authors certify that there is no conflict of interest with any financial organization regarding the material discussed in the manuscript. Article first published online: November 27, 2018. - Manuscript accepted: October 30, 2018. - Manuscript received: October 18, 2018. 